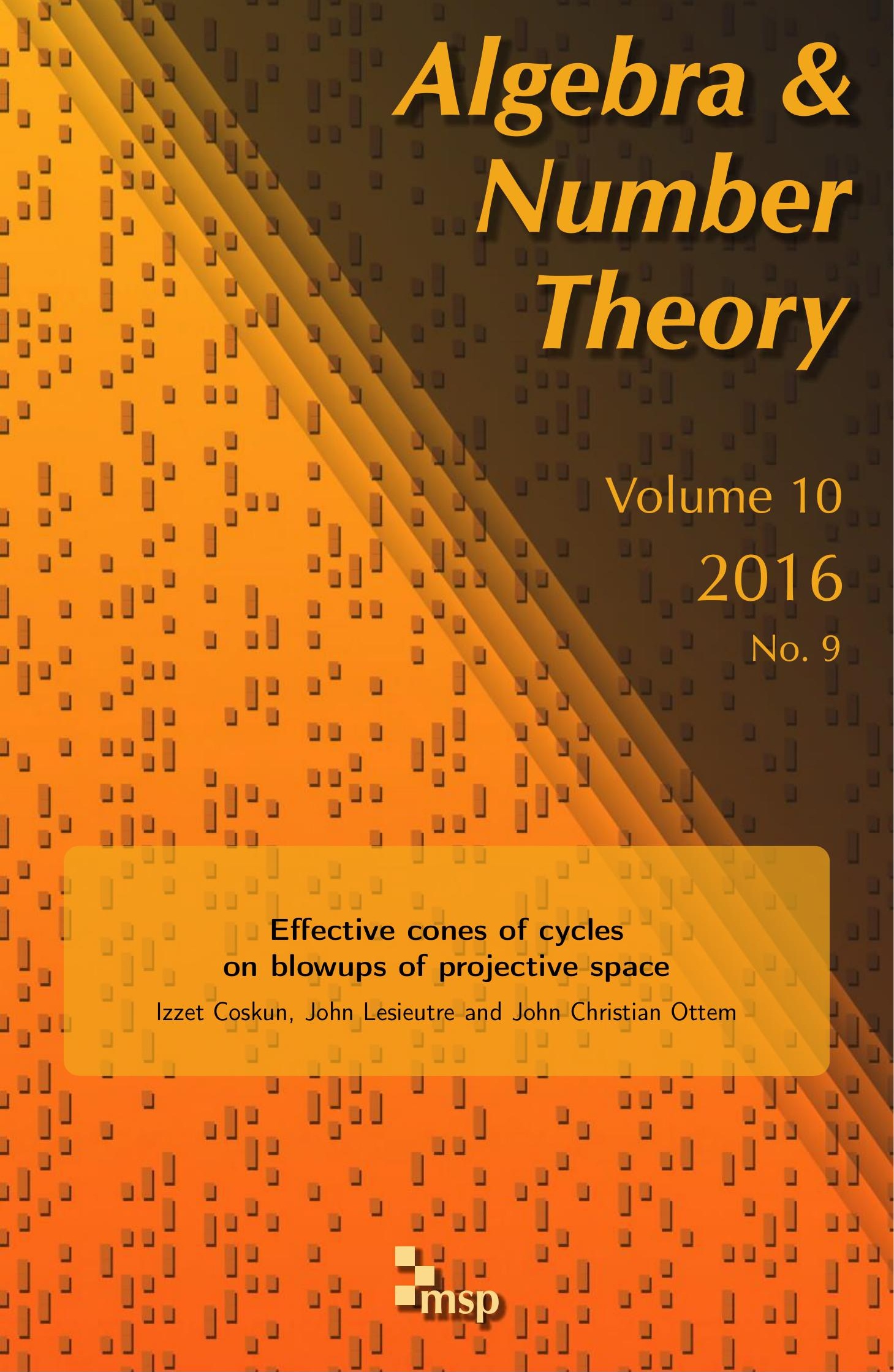




\title{
Effective cones of cycles on blowups of projective space
}

\author{
Izzet Coskun, John Lesieutre and John Christian Ottem
}

\begin{abstract}
In this paper we study the cones of higher codimension (pseudo)effective cycles on point blowups of projective space. We determine bounds on the number of points for which these cones are generated by the classes of linear cycles and for which these cones are finitely generated. Surprisingly, we discover that for (very) general points the higher codimension cones behave better than the cones of divisors. For example, for the blowup $X_{r}^{n}$ of $\mathbb{P}^{n}, n>4$ at $r$ very general points, the cone of divisors is not finitely generated as soon as $r>n+3$, whereas the cone of curves is generated by the classes of lines if $r \leq 2^{n}$. In fact, if $X_{r}^{n}$ is a Mori dream space then all the effective cones of cycles on $X_{r}^{n}$ are finitely generated.
\end{abstract}

\section{Introduction}

In recent years, the theory of cones of cycles of higher codimension has been the subject of increasing attention [Chen and Coskun 2015; Debarre et al. 2011; 2013; Fulger and Lehmann 2014a; 2014b]. However, these cones have been computed only for a very small number of examples, mainly because the current theory is hard to apply in practice. The goal of this paper is to provide some much-needed examples.

Let $\Gamma$ be a set of $r$ distinct points on $\mathbb{P}^{n}$. Let $X_{\Gamma}^{n}$ denote the blowup of $\mathbb{P}^{n}$ along $\Gamma$. When $\Gamma$ is a set of $r$ very general points, we denote $X_{\Gamma}^{n}$ by $X_{r}^{n}$. For a smooth variety $Y$, we write $\overline{\operatorname{Eff}}^{k}(Y)$ for the pseudoeffective cone of codimension- $k$ cycles on $Y$, and $\overline{\operatorname{Eff}}_{k}(Y)$ for the pseudoeffective cone of dimension- $k$ cycles. In this paper, we study the cones $\overline{\operatorname{Eff}}_{k}\left(X_{\Gamma}^{n}\right)$ when the points of $\Gamma$ are either in linearly general or very general position. We also investigate the cones when $\Gamma$ contains points in certain special configurations.

During the preparation of this article the first author was partially supported by the NSF CAREER grant DMS-0950951535 and NSF grant DMS-1500031, the second author was partially supported by an NSF RTG grant, and the third author was supported by RCN grant 250104.

MSC2010: primary 14C25, 14C99; secondary 14E07, 14E30, 14M07, 14N99.

Keywords: Cones of effective cycles, higher codimension cycles, blowups of projective space, Mori dream space. 
Cones of positive divisors on $X_{\Gamma}^{n}$ provide an important source of examples in the study of positivity. These cones are particularly attractive since they have concrete interpretations in terms of subvarieties of projective space, yet still have very complicated structure. However, even the cones of divisors on blowups of $\mathbb{P}^{2}$ at 10 or more points are far from well-understood, and several basic questions remain open, including the Nagata conjecture [1959] and the Segre-HarbourneGimigliano-Hirschowitz (SHGH) conjecture [Gimigliano 1987; Harbourne 1986; Hirschowitz 1989]. We expect the cones of higher codimension cycles on $X_{\Gamma}^{n}$ to be an equally rich source of examples.

Surprisingly, these cones are simpler than one might expect. Effective cones of low-dimensional cycles are generated by the classes of linear spaces for $r$ well into the range for which $X_{r}^{n}$ ceases to be a Mori dream space. For example, $\overline{\operatorname{Eff}}_{1}\left(X_{r}^{n}\right)$ is generated by classes of lines for $r \leq 2^{n}$ even though $\overline{\operatorname{Eff}}^{1}\left(X_{r}^{n}\right)$ is not finitely generated for $r \geq n+4$ when $n \geq 5$. We now describe our results in greater detail.

Definition 1.1. We say that $\overline{\operatorname{Eff}}_{k}\left(X_{\Gamma}^{n}\right)$ is linearly generated if it is the cone spanned by the classes of $k$-dimensional linear spaces in the exceptional divisors and the strict transforms of $k$-dimensional linear subspaces of $\mathbb{P}^{n}$, possibly passing through the points of $\Gamma$. We say $\overline{\operatorname{Eff}}_{k}\left(X_{\Gamma}^{n}\right)$ is finitely generated if it is a rational polyhedral cone.

Theorem 3.1. Let $\Gamma$ be a set of $r$ points in $\mathbb{P}^{n}$ in linearly general position. If $r \leq \max (n+2, n+n / k)$, then $\overline{\operatorname{Eff}}_{k}\left(X_{\Gamma}^{n}\right)$ is linearly generated.

There exist configurations of $2 n+2-k$ points in linearly general position in $\mathbb{P}^{n}$ for which $\overline{\operatorname{Eff}}_{k}\left(X_{\Gamma}^{n}\right)$ is not linearly generated (see Example 3.4). In particular, Theorem 3.1 is sharp for 1-cycles. We expect that this bound can be improved to $r \leq 2 n+1-k$, and prove this in the case that $\Gamma$ is a very general configuration of points (Theorem 4.5). We obtain the following consequence.

Corollary 4.7. If $X_{r}^{n}$ is a Mori dream space, then $\overline{\operatorname{Eff}}_{k}\left(X_{r}^{n}\right)$ is finitely generated.

In general, Mori dream spaces may have effective cones of intermediate dimensional cycles which are not finitely generated; the corollary shows that this does not happen for blowups of $\mathbb{P}^{n}$. A good example is [Debarre et al. 2011, Example 6.10], attributed to Tschinkel. Let $X_{b}$ be the blowup of $\mathbb{P}^{4}$ along a smooth quartic K3 surface $Y_{b} \subset \mathbb{P}^{3} \subset \mathbb{P}^{4}$. Then $X_{b}$ is Fano, hence, by [Birkar et al. 2010], a Mori dream space. On the other hand, $\overline{\operatorname{Eff}}_{2}\left(X_{b}\right)$ has infinitely many extremal rays when $\overline{\mathrm{Eff}}_{1}\left(Y_{b}\right)$ does. Quartic K3 surfaces may have infinitely many $(-2)$-curves or even a round cone of curves. This example also shows that the property of having finitely generated higher codimension cones can fail countably many times in a family.

The bounds can be exponentially improved (at least for 1-cycles) if we assume that $\Gamma$ is a set of very general points.

Proposition 4.1. The cone $\overline{\operatorname{Eff}}_{1}\left(X_{r}^{n}\right)$ is linearly generated if and only if $r \leq 2^{n}$. 
As a consequence of Proposition 4.1, we conclude that $\overline{\operatorname{Eff}}_{k}\left(X_{r}^{n}\right)$ is not linearly generated if $r \geq 2^{n-k+1}+k$ (Corollary 4.2). This specializes to the fact that the cone of divisors of $X_{r}^{n}$ is not linearly generated as soon as $r>n+2$ (see Theorem 2.7).

Mukai [2004] shows that the cone of divisors of $X_{r}^{n}$ is not finitely generated if $r \geq n+4$ and $n \geq 5$ (one needs $r \geq 9$ for $n=2$ or 4 , and $r \geq 8$ for $n=3$ ). Mukai explicitly constructs infinitely many extremal divisors on $\overline{\overline{E f f}}^{1}\left(X_{r}^{n}\right)$ as the orbit of one of the exceptional divisors under the action of Cremona transformations. However, in higher codimensions it is more difficult to prove that the corresponding cones become infinite.

Many questions about cones of higher codimension cycles appear to be intractable, quickly reducing to difficult questions about cones of divisors. For example, the interesting part of the cone of curves of $\mathbb{P}^{3}$ blown up at 9 points is given by curves lying on the unique quadric $Q$ through the 9 points. The blowup of $Q$ is isomorphic to the blowup of $\mathbb{P}^{2}$ at 10 points, and the curves which are extremal on $X_{9}^{3}$ are certain $K_{Q}$-positive ones contained in $Q$. Hence understanding $\overline{\operatorname{Eff}}_{1}\left(X_{9}^{3}\right)$ requires understanding the $K_{X_{10}^{2}}$-positive part of $\overline{\mathrm{Eff}}_{1}\left(X_{10}^{2}\right)$, running immediately into the SHGH conjecture (see Conjecture 5.1). We are able to show this nonfiniteness only for cones of codimension- 2 cycles, and then assuming the SHGH conjecture on the cone of curves of $\mathbb{P}^{2}$ blown up at 10 points.

Corollary 5.7. Assume the SHGH conjecture holds for blowups of $\mathbb{P}^{2}$ at 10 points, then $\overline{\mathrm{Eff}}^{2}\left(X_{r}^{n}\right)$ is not finitely generated if $r \geq n+6$ and $n \geq 3$.

Finally, in the last section, we compute $\overline{\operatorname{Eff}}_{k}\left(X_{\Gamma}^{n}\right)$ when $\Gamma$ is a set of points in certain special positions. Using these computations, we show that linear and finite generation of $\overline{\mathrm{Eff}}_{k}\left(X_{\Gamma}^{n}\right)$ are neither open nor closed in families (see Corollaries 6.6 and 6.7). This generalizes analogous jumping behavior exhibited for divisors and Mori dream spaces to all codimensions.

The organization of the paper. In Section 2, we collect basic facts concerning the cohomology of $X_{\Gamma}^{n}$, cones of divisors, the action of Cremona transformations, and some preliminary lemmas. In Section 3, we prove Theorem 3.1 and study the linear generation of the cones $\overline{\operatorname{Eff}}_{k}\left(X_{\Gamma}^{n}\right)$ when $\Gamma$ is a linearly general set of points. In Section 4, we study the linear generation of the cones $\overline{\operatorname{Eff}}_{k}\left(X_{r}^{n}\right)$. In Section 5, we prove that $\overline{\operatorname{Eff}}^{2}\left(X_{r}^{n}\right)$ is not finitely generated for $r \geq n+6$ assuming the SHGH conjecture. In Section 6, we discuss the cones $X_{\Gamma}^{n}$ when $\Gamma$ contains points in certain special configurations and study the variation of $\overline{\operatorname{Eff}}_{k}\left(X_{\Gamma}^{n}\right)$ in families.

\section{Preliminaries}

In this section, we recall basic facts about the cohomology of $X_{\Gamma}^{n}$ and cones of codimension- 1 cycles. We will work over the complex numbers $\mathbb{C}$. 
The cohomology of $\boldsymbol{X}_{\Gamma}^{\boldsymbol{n}}$. Let $\Gamma$ be a set of $r$ points $p_{1}, \ldots, p_{r}$ in $\mathbb{P}^{n}$, and let

$$
\pi: X_{\Gamma}^{n}=\mathrm{Bl}_{\Gamma} \mathbb{P}^{n} \rightarrow \mathbb{P}^{n}
$$

denote the blowup of $\mathbb{P}^{n}$ along $\Gamma$. Let $H$ denote the pullback of the hyperplane class and let $E_{i}$ denote the class of the exceptional divisor over $p_{i}$. The exceptional divisor $E_{i}$ is isomorphic to $\mathbb{P}^{n-1}$ and $\mathcal{O}_{E_{i}}\left(E_{i}\right) \cong \mathcal{O}_{\mathbb{P}^{n-1}}(-1)$. Consequently, we have the following intersection formulas:

$$
H^{n}=(-1)^{n-1} E_{i}^{n}=1, \quad H \cdot E_{i}=0, \quad E_{i} \cdot E_{j}=0, i \neq j .
$$

Notation 2.1. In order to simplify notation, we make the convention that $H_{k}$ is the class of a $k$-dimensional linear space in $\mathbb{P}^{n}$ and $E_{i, k}$ is the class of a $k$-dimensional linear space contained in the exceptional divisor $E_{i}$. We then have the relations

$$
H^{n-k}=H_{k}, \quad(-1)^{n-k+1} E_{i}^{n-k}=E_{i, k}, \quad E_{i} \cdot E_{i, k}=-E_{i, k-1} .
$$

On $X_{\Gamma}^{n}$ homological, numerical, and rational equivalence coincide. For $0<k<n$, we write $N_{k}\left(X_{\Gamma}^{n}\right)$ for the $\mathbb{R}$-vector space of $k$-dimensional cycles on $X_{\Gamma}^{n}$, modulo numerical equivalence. Dually, $N^{k}\left(X_{\Gamma}^{n}\right)$ denotes the space of codimension- $k$ cycles modulo numerical equivalence. They are both $(r+1)$-dimensional vector spaces.

A class in $N_{k}\left(X_{\Gamma}^{n}\right)$ is said to be pseudoeffective if it is the limit of classes of effective cycles. We write $\overline{\operatorname{Eff}}_{k}\left(X_{\Gamma}^{n}\right)$ for the closed convex cone in $N_{k}\left(X_{\Gamma}^{n}\right)$ containing pseudoeffective classes. If $V$ is an (irreducible) $k$-dimensional subvariety of $X_{\Gamma}^{n}$, we write [V] for the class of $V$ in $N_{k}\left(X_{\Gamma}^{n}\right)$, although when confusion seems unlikely we omit the brackets.

A set of points in $\mathbb{P}^{n}$ is said to be linearly general if no $k+2$ points are contained in a linear subspace $\mathbb{P}^{k} \subset \mathbb{P}^{n}$ for $1 \leq k \leq n-1$. A claim holds for a very general configuration of points if it holds for all points in the complement of a countable union of proper configurations of points.

Convention 2.2. It is occasionally useful to compare the cones $\overline{\operatorname{Eff}}_{k}\left(X_{\Gamma}^{n}\right)$ and $\overline{\operatorname{Eff}}_{k}\left(X_{\Delta}^{m}\right)$, where $X_{\Gamma}^{n}$ and $X_{\Delta}^{m}$ are the blowups of $\mathbb{P}^{n}$ and $\mathbb{P}^{m}$ along sets of points $\Gamma$ and $\Delta$, respectively. If $n>k$, we can identify $N_{k}\left(X_{\Gamma}^{n}\right)$ with the abstract vector space spanned by $H_{k}$ and $E_{i, k}$ for $1 \leq i \leq r$, irrespective of $n$ and $\Gamma$ provided that $\Gamma$ has cardinality $r$. We can thus view the cones $\overline{\operatorname{Eff}}_{k}\left(X_{\Gamma}^{n}\right)$ as cones in the same abstract vector space and compare the effective cones of different blowups after this identification. In the rest of the paper, we will do so without further comment.

We will often use the following easy lemma implicitly.

Lemma 2.3. Let $Y \subset X_{\Gamma}^{n}$ be a k-dimensional subvariety:

(1) If $Y \subset E_{i}$ for some $1 \leq i \leq r$, then $[Y]=b_{i} E_{i, k}$ for $b_{i}>0$.

(2) Otherwise, $[Y]=a H_{k}-\sum_{i=1}^{r} b_{i} E_{i, k}$ with $a \geq b_{i} \geq 0$. The coefficient $b_{i}$ is equal to the multiplicity of $\pi(Y)$ in $\mathbb{P}^{n}$ at the point $p_{i}$. 
Proof. If $Y \subset E_{i}$, then $Y$ is a subvariety of $E_{i} \cong \mathbb{P}^{n-1}$. Hence its class is a positive multiple of the class of a $k$-dimensional linear space. The linear system $H-E_{i}$ defines the projection from the point $p_{i}$ and is a basepoint-free linear system. Hence the intersection of $k$ general members of $H-E_{i}$ with $Y$ is either empty or finitely many points. Therefore, $\left(H-E_{i}\right)^{k} \cdot[Y]=a-b_{i} \geq 0$. Similarly, the intersection $Y \cap E_{i}$ is a (possibly empty) effective cycle of dimension $k-1$ contained in $E_{i}$. Hence by the first part of the lemma, $b_{i} \geq 0$. That $b_{i}$ in fact coincides with the multiplicity is [Fulton 1998, Corollary 6.7.1].

The cones $\overline{\mathrm{Eff}}_{k}\left(X_{\Gamma}^{n}\right)$ satisfy a basic semicontinuity property under specialization.

Lemma 2.4. Suppose that $V \subset \mathbb{P}^{n} \times T$ is a closed subvariety, flat over $T$, with fibers of dimension $k$, and let $p: T \rightarrow \mathbb{P}^{n}$ be a section. Then $\operatorname{mult}_{p(t)}\left(V_{t}\right)$ is an upper semicontinuous function on $T$.

Proof. It suffices to prove this in the case that $T$ has dimension 1. Let $\pi: Y \rightarrow \mathbb{P}^{n} \times T$ be the blowup along $p(T)$, with exceptional divisor $E$, and let $\widetilde{V}$ be the strict transform of $V$ on $Y$. Since $\widetilde{V}$ is irreducible and dominates $T$, this family is flat. The intersection of a flat family of cycles with a Cartier divisor is constant in $t$ [Fulton 1998, Proposition 10.2.1], and so $(-1)^{k+1} E^{k} \cdot \widetilde{V}_{t}$ is independent of $t$.

The general fiber $\widetilde{V}_{t}$ is irreducible, but a special fiber $\widetilde{V}_{0}$ may have additional components in the exceptional divisor $E_{0}$. Write $\widetilde{V}_{0}=V^{0} \cup \bigcup_{i} W_{i}$, where the $W_{i}$ are contained in $E_{0}$. Then $(-1)^{k+1} E^{k} \cdot V^{0}=\operatorname{mult}_{p(0)} V_{0}$. The class $(-1)^{k+1} E_{0}^{k}$ is a linear space in $E_{0}$, and so $(-1)^{k+1} E_{0}^{k} \cdot W_{i} \geq 0$. This shows that mult $p_{p(0)} V_{0} \geq$ $(-1)^{k+1} E^{k} \cdot \widetilde{V}_{t}=$ mult $_{p(t)} V_{t}$, and so the multiplicity is upper semicontinuous.

Corollary 2.5. Let $\Gamma$ be a configuration of $r$ distinct points on $\mathbb{P}^{n}$. Then

$$
\overline{\operatorname{Eff}}_{k}\left(X_{r}^{n}\right) \subseteq \overline{\operatorname{Eff}}_{k}\left(X_{\Gamma}^{n}\right) .
$$

Proof. Let $\Gamma_{t}$ be a very general one-parameter family of configurations of points in $\mathbb{P}^{n}$ with $\Gamma_{0}=\Gamma$. If a $k$-cycle class $W$ is effective for very general $T$, then by a Hilbert scheme argument there exists a flat family $V_{t} \subset \mathrm{Bl}_{\Gamma_{t}} \mathbb{P}^{n}$ over $T$ with $\left[V_{t}\right]=W$ for general $T$. Since the multiplicity of $W_{t}$ can only increase at $t=0$ by Lemma 2.4, the class $W$ is also effective on $X_{\Gamma}$.

Cones. Taking cones will be a useful method to generate interesting cycles. Let $\Gamma^{\prime}$ be a very general configuration of $r+1$ points $p_{0}^{\prime}, \ldots, p_{r}^{\prime}$ in $\mathbb{P}^{n+1}$. The projection of the points $p_{1}^{\prime}, \ldots, p_{r}^{\prime}$ from $p_{0}^{\prime}$ is then a set $\Gamma$ of $r$ very general points $p_{1}, \ldots, p_{r}$ in $\mathbb{P}^{n}$. Suppose that $V$ is a $k$-cycle on $X_{\Gamma}^{n}$, with class $a H_{k}-\sum_{i=1}^{r} b_{i} E_{i, k}$. The image of $V$ in $\mathbb{P}^{n}$ has degree $a$ and multiplicity $b_{i}$ at the points of $\Gamma$. We may form the cone $C V$ over $V$ inside $\mathbb{P}^{n+1}$ with vertex at $p_{0}^{\prime}$. This is a $(k+1)$-dimensional variety of degree $a$. It has multiplicity $a$ at the cone point and multiplicity $b_{i}$ along the lines spanned by $p_{i}$ and $p_{i}^{\prime}$ for $1 \leq i \leq r$. In particular, the cycle $C V$ has degree $a$ 
and multiplicities $a, b_{1}, \ldots, b_{r}$ at the points of $\Gamma^{\prime}$. Its proper transform has class $a H_{k+1}-a E_{0, k+1}-\sum_{i=1}^{r} b_{i} E_{i, k+1}$.

We define a map $C: N_{k}\left(X_{r}^{n}\right) \rightarrow N_{k+1}\left(X_{r+1}^{n+1}\right)$ by $C\left(H_{k}\right)=H_{k+1}-E_{0, k+1}$ and $C\left(E_{i, k}\right)=E_{i, k+1}$. With this definition, $C([V])$ is the class of the cone over $V$ with vertex $p_{0}^{\prime}$, and so $C\left(\overline{\operatorname{Eff}}_{k}\left(X_{r}^{n}\right)\right) \subseteq \overline{\operatorname{Eff}}_{k+1}\left(X_{r+1}^{n+1}\right)$ with respect to the identification discussed in Convention 2.2.

The following computation of a dual cone will be useful on a number of occasions.

Lemma 2.6. Suppose that $\mathbf{v}=\left(a,-b_{1}, \ldots,-b_{r}\right) \in \mathbb{Z}^{r+1}$ is a vector satisfying

(1) $a, b_{i} \geq 0$,

(2) $a \geq b_{i}$ for every $i$,

(3) $n a \geq \sum_{i=1}^{r} b_{i}$.

Then $\mathbf{v}$ is a positive linear combination of the vectors $e_{i}$, for $1 \leq i \leq r$, and $h_{I}=e_{0}-\sum_{i \in I} e_{i}$ with $|I| \leq n$. When $r \geq n$, we may assume each term has $|I|=n$. Proof. Note first that the vectors $h_{I}=e_{0}-\sum_{i \in I} e_{i}$ with $|I|<n$ are positive linear combinations of the given vectors. We now proceed by induction on $a$. The case $a=1$ is clear since by (2) $a \geq b_{i}$ for each $i$, each $b_{i}$ is either 0 or 1 . By (3) there are at most $n$ nonzero $b_{i}$, and the vector is of the form claimed.

Suppose that $a>1$. Let $J$ be the set of indices $i$ such that $b_{i}>0$, and let $j=\min (n,|J|)$. Let $I$ be a set of $j$ indices $\left\{i_{1}, \ldots, i_{j}\right\}$ such that $b_{i_{1}} \geq \cdots \geq b_{i_{j}} \geq b_{i}$ for any $i \notin I$. Then the vector $h_{I}$ is a nonnegative linear combination of the given vectors. Set $v^{\prime}=\left(a^{\prime},-b_{1}^{\prime}, \ldots,-b_{r}^{\prime}\right)=\mathbf{v}-h_{I}$. If $j \geq n$, then $v^{\prime}$ still satisfies all of the inequalities in question since $a^{\prime}=a-1$ and $\sum b_{i}^{\prime}=\sum b_{i}-n$. If $j<n$, then in view of inequality (2), the inequality (3) can be improved to $j a \geq \sum_{i=1}^{r} b_{i}$. Then $v^{\prime}$ satisfies these improved inequalities. This completes the proof by induction on $a$.

Lemma 2.6 implies that the cone $\overline{\operatorname{Eff}}_{k}\left(X_{r}^{n}\right)$ is linearly generated if and only if the class $(k+1) H_{n-k}-\sum_{i=1}^{r} E_{i, n-k}$ is nef.

The codimension-1 cones and Cremona actions. We are primarily interested in the question of when the cones of cycles on $X_{\Gamma}^{n}$ are linearly or finitely generated. For cones of divisors, the answers to these questions were worked out by Castravet and Tevelev [2006] and Mukai [2004].

Theorem 2.7 [Castravet and Tevelev 2006; Mukai 2004]. Let $\Gamma$ be a set of $r$ very general points in $\mathbb{P}^{n}$. The cone $\overline{\mathrm{Eff}}^{1}\left(X_{\Gamma}^{n}\right)$ is linearly generated if and only if $r \leq n+2$, and finitely generated if and only if

(1) $n=2$ and $r \leq 8$,

(2) $n=3$ and $r \leq 7$,

(3) $n=4$ and $r \leq 8$,

(4) $n \geq 5$ and $r \leq n+3$. 
The characterization of cases when the effective cone of divisors is finitely generated is based on the study of the action of Cremona transformations on the pseudoeffective cone. The Coxeter group $W$ corresponding to a $T$-shaped Dynkin diagram of type $T_{2, n+1, r-n-1}$ acts on $N^{1}\left(X_{\Gamma}^{n}\right)$ and preserves the pseudoeffective cone $\overline{\mathrm{Eff}}^{1}\left(X_{\Gamma}^{n}\right)$. This is an infinite group if $\frac{1}{2}+\frac{1}{n+1}+\frac{1}{r-n-1}<1$, which happens as soon as $n \geq 5$ and $r \geq n+4$. (When $n=2$ or 4 , we need $r \geq 9$; while when $n=3$, we require $r \geq 8$ ). The orbit of a single exceptional divisor class gives an infinite set of divisors spanning other extremal rays. For details on this group action, we refer to [Dolgachev 2011] (see also [Coble 1982]).

Unfortunately, there does not seem to be a simple way to use the Cremona action to understand cones of cycles of higher codimension. The standard Cremona involution acts on $X_{r}^{n}$ by a map with codimension 2 indeterminacy, so it does not define an action preserving the cone $\overline{\mathrm{Eff}}^{k}\left(X_{r}^{n}\right)$ for any $k>1$. For example, suppose that $L$ is a line through two blown up points. The class of $L$ defines an extremal ray on $\overline{\mathrm{Eff}}_{1}\left(X_{r}^{n}\right)$. The strict transform of $L$ under a Cremona transformation centered at $n+1$ other points is a rational normal curve in $\mathbb{P}^{n}$ passing through $n+3$ points. If $n \geq 3$, this is no longer an extremal ray on $\overline{\operatorname{Eff}}_{1}\left(X_{r}^{n}\right)$, since it is in the interior of the subcone generated by classes of lines through 2 of the $n+3$ points.

One might attempt to construct interesting codimension-2 cycles on $X_{n}^{r}$ by taking the intersections of a fixed divisor with an infinite sequence of $(-1)$-divisors (i.e., divisors in the orbit of $E_{i}$ under the action of $W$ ) of increasing degree. However, the next lemma shows that the intersection of a $(-1)$-divisor with any other effective divisor on $X_{r}^{n}$ is in the span of the classes of codimension-2 linear cycles.

Lemma 2.8. Suppose that $D_{1}$ is a (-1)-divisor and that $D_{2}$ is an irreducible effective divisor distinct from $D_{1}$. Then $\left[D_{1} \cap D_{2}\right]$ is in the span of linear codimension-2 cycles.

Proof. Consider the pairing on $N^{1}\left(X_{r}^{n}\right)$ defined by $(H, H)=n-1,\left(H, E_{i}\right)=0$, $\left(E_{i}, E_{i}\right)=-1$, and $\left(E_{i}, E_{j}\right)=0$ if $i \neq j$. This pairing is invariant under the action of $W$ on $N^{1}\left(X_{r}^{n}\right)$ [Mukai 2004; Dolgachev 2011].

We first show that $\left(D_{1}, D_{2}\right) \geq 0$. Since the pairing $($,$) is invariant under the$ action of $W$ on $N^{1}\left(X_{r}^{n}\right)$, we may apply a suitable element of $W$ and assume that $D_{1}=E_{1}$ is an exceptional divisor. If $D_{2}=E_{j}$ is an exceptional divisor different from $E_{1}$, then $\left(D_{1}, D_{2}\right)=0$. Otherwise, $\left[D_{2}\right]=a H-\sum_{i=1}^{r} b_{i} E_{i}$, with $b_{i} \geq 0$, in which case $\left(D_{1}, D_{2}\right)=b_{i} \geq 0$.

For the second part, write $D_{1}=a H-\sum_{i=1}^{r} b_{i} E_{i}$ and $D_{2}=c H-\sum_{i=1}^{r} d_{i} E_{i}$. That $\left(D_{1}, D_{2}\right) \geq 0$ yields

$$
(n-1) a c \geq \sum_{i=1}^{r} b_{i} d_{i}
$$


By Lemma 2.6, this means that the codimension-2 cycle $\left[D_{1} \cap D_{2}\right]=a c H-$ $\sum_{i=1}^{r} b_{i} d_{i}$ is contained in the span of linear cycles.

Easy lemmas. Here we collect a couple of geometric lemmas that we will use repeatedly.

Lemma 2.9. Suppose that $E$ is an effective divisor and that $P$ is a nef divisor. If $Y$ is an irreducible, effective variety of dimension $k$ which is not contained in $E$, then $P^{k-1} \cdot E \cdot Y \geq 0$.

Proof. The intersection $E \cdot Y$ is a (possibly empty) cycle of dimension $k-1$ by assumption. Since $P$ is nef, it follows that $P^{k-1} \cdot E \cdot Y \geq 0$.

Lemma 2.10. Let $Y \subset X_{\Gamma}^{n}$ be an irreducible variety of dimension $k$, not contained in any exceptional divisor $E_{i}$, with class $a H_{k}-\sum_{i=1}^{e} b_{i} E_{i, k}$. If $b_{i}+b_{j}>$ a for two indices $i \neq j$, then $Y$ contains the line through $p_{i}$ and $p_{j}$ with multiplicity at least $b_{i}+b_{j}-a$.

Proof. The base locus of the linear system $\left|H-E_{i}-E_{j}\right|$ is the line $l_{i, j}$ spanned by $p_{i}$ and $p_{j}$. Consequently, the intersection $\left(H-E_{i}-E_{j}\right)^{k-1} \cdot Y$ is an effective 1 -cycle $Z$. Express

$$
Z=\alpha l_{i, j}+u,
$$

where $u$ is a 1 -cycle not containing $l_{i, j}$. Since

$$
-\alpha \leq\left(H-E_{i}-E_{j}\right) \cdot Z=a-b_{i}-b_{j}<0,
$$

we conclude that $\alpha \geq b_{i}+b_{j}-a$. Hence $Y$ must have multiplicity at least $b_{i}+b_{j}-a$ at every point of $l_{i, j}$.

\section{Points in linearly general position}

In this section, we study $\overline{\operatorname{Eff}}_{k}\left(X_{\Gamma}^{n}\right)$ when the cardinality of $\Gamma$ is small and the points of $\Gamma$ are in linearly general position. Our main theorem is the following.

Theorem 3.1. Let $\Gamma$ be a set of $r$ points in $\mathbb{P}^{n}$ in linearly general position. If

$$
r \leq \max \left(n+2, n+\frac{n}{k}\right),
$$

then $\overline{\operatorname{Eff}}_{k}\left(X_{\Gamma}^{n}\right)$ is linearly generated.

The proof will be by induction on $k$ and $n$. We first single out the case $k=1$.

Lemma 3.2. Let $\Gamma$ be a set of $r \leq 2 n$ points in $\mathbb{P}^{n}$ in linearly general position. Then $\overline{\mathrm{Eff}}_{1}\left(X_{\Gamma}^{n}\right)$ is linearly generated. 
Proof. Let $B$ be an irreducible curve. By Lemma 2.3, we may assume that $B$ is not contained in any of the exceptional divisors and has class $a H_{1}-\sum_{i=1}^{r} b_{i} E_{i, 1}$ with $a \geq b_{i} \geq 0$. Any $r \leq 2 n$ points in linearly general position are cut out by quadrics [Harris 1995, Lecture 1]. Consequently, there is a quadric whose proper transform has class $[Q]=2 H-\sum_{i=1}^{r} E_{i}$ in $X_{\Gamma}^{n}$ and does not contain $B$. Hence $B$ has nonnegative intersection with $Q$ and satisfies $2 a \geq \sum_{i=1}^{r} b_{i}$. By Lemma 2.6, the class of $B$ is spanned by the classes of lines.

Next, we study the case when $r \leq n+1$. In this case, $X_{\Gamma}^{n}$ is toric and the effective cones are generated by torus-invariant cycles (see e.g., [Li 2015, Proposition 3.1]). For the reader's convenience we will give a simple independent proof.

Lemma 3.3. Let $\Gamma$ be a set of $r \leq n+1$ linearly general points in $\mathbb{P}^{n}$. The cone $\overline{\mathrm{Eff}}_{k}\left(X_{\Gamma}^{n}\right)$ is linearly generated for any $k$.

Proof. Let $\Gamma^{\prime} \subset \Gamma$ be two sets with cardinality $r$ and $n+1$, respectively. Then the proper transform of any effective cycle in $X_{\Gamma^{\prime}}^{n}$ is an effective cycle in $X_{\Gamma}^{n}$. Consequently, if $\overline{\operatorname{Eff}}_{k}\left(X_{\Gamma}^{n}\right)$ is linearly generated, then $\overline{\operatorname{Eff}}_{k}\left(X_{\Gamma^{\prime}}^{n}\right)$ is also linearly generated. Hence, without loss of generality, we may assume that $r=n+1$. Let $Y$ be an irreducible $k$-dimensional variety in $X_{\Gamma}^{n}$ with class

$$
[Y]=a H_{k}-\sum_{i=1}^{n+1} b_{i} E_{i, k} .
$$

By Lemma 2.3, we may assume that $Y$ is not contained in an exceptional divisor and that $a \geq b_{i} \geq 0$. We proceed by induction on $k$ and $n$. After reordering the points, we may assume $b_{1} \geq b_{2} \geq \cdots \geq b_{n+1}$. Let $L$ be the proper transform of the $\mathbb{P}^{n-1}$ spanned by the first $n$ points. First, suppose $Y$ is contained in $L$. Since $L$ is isomorphic to the blowup of $\mathbb{P}^{n-1}$ in $n$ points, by induction on $n$ with base case Theorem 2.7, we conclude that the class of $Y$ is in the span of linear spaces. Otherwise, $Y \cap L$ is an effective cycle of dimension $k-1$ in $L$. Write $H_{L, k-1}$ and $E_{L, i, k-1}$ for the restriction of $H_{k}$ to $L$ and the $(k-1)$-dimensional linear space in the exceptional divisor $E_{L, i}$ of the blowup of $L$ at $p_{i}$. Then we have

$$
[Y \cap L]=a H_{L, k-1}-\sum_{i=1}^{n} b_{i}^{\prime} E_{L, i, k-1},
$$

with $b_{i}^{\prime} \geq b_{i}$. By induction on $n$ with base case Lemma 3.2, $Y \cap L$ is in the span of linear spaces. In particular, $k a \geq \sum_{i=1}^{n} b_{i}^{\prime}$. Hence $(k+1) a \geq \sum_{i=1}^{n+1} b_{i}$. By Lemma 2.6, the class of $Y$ is in the span of linear spaces. This concludes the proof.

We can now complete the proof of Theorem 3.1.

Proof of Theorem 3.1. We preserve the notation from the proof of Lemma 3.3 and argue similarly. It suffices to check the result in the case $r=\max (n+2, n+n / k)$. 
Suppose that $Y$ is an irreducible $k$-dimensional variety on $X_{\Gamma}^{n}$ with class

$$
[Y]=a H_{k}-\sum_{i=1}^{r} b_{i} E_{i, k}
$$

We may assume that $Y$ is not contained in an exceptional divisor and, by reordering the points, we have that

$$
a \geq b_{1} \geq \cdots \geq b_{r} \geq 0 .
$$

Let $L$ be the $\mathbb{P}^{n-1}$ passing through the points $p_{1}, \ldots, p_{n}$. If $Y$ is contained in $L$, then its class is linearly generated by Lemma 3.3. Otherwise, $Y \cap L$ is an effective cycle of dimension $k-1$ with class

$$
[Y \cap L]=a H_{L, k-1}-\sum_{i=1}^{n} b_{i}^{\prime} E_{L, i, k-1},
$$

with $b_{i}^{\prime} \geq b_{i}$. This class and hence $a H_{L, k-1}-\sum_{i=1}^{n} b_{i} E_{L, i, k-1}$ is linearly generated by Lemma 3.3. Therefore, it can be written as a combination of linear classes $H_{L, k-1}-\sum_{|I|=k} E_{L, i, k-1}$ and $E_{L, i, k-1}$,

$$
\sum_{j=1}^{a} \alpha_{j}\left(H_{L, k-1}-\sum_{|I|=k} E_{L, i, k-1}\right)+\sum_{j=1}^{n} \beta_{j} E_{L, j, k-1} .
$$

Each of the classes in this sum is effective, with those on the left the classes of $\mathbb{P}^{k-1}$ through $k$ of the points in $L$. By taking cones over these classes, we obtain a $\mathbb{P}^{k}$ on $X$, passing through an additional one of the points $p_{i}$ with $i>n$. Since there are $a$ planes available, if $\sum_{i=n+1}^{r} b_{i} \leq a$, the class $Y$ can be expressed as a sum of linear cycles.

Observe that

$$
a k \geq \sum_{i=1}^{n} b_{i} \geq n b_{n}, \quad \text { and so } \quad b_{j} \leq b_{n} \leq \frac{a k}{n} \text { for } j \geq n .
$$

This implies that if $(r-n) k / n \leq 1$ or equivalently if $r \leq n+n / k$, the classes of all effective cycles are in the span of the classes of linear spaces.

If $k \leq n / 2$, then $n+2 \leq n+n / k$ and the theorem is proved. If $k>n / 2$, then $n+1<n+n / k<n+2$ and we need to settle the case $r=n+2$. There is a rational normal curve through any $n+3$ points in linearly general position in $\mathbb{P}^{n}$ [Harris 1995, Lecture 1]. Consequently, given an effective divisor $D$, there exists a rational normal curve $C$ containing the points but not contained in $D$. Hence $C \cdot D \geq 0$ and all effective divisors satisfy $n a \geq \sum_{i=1}^{n+2} b_{i}$. We recover the linear generation result of Theorem 2.7. By Lemma 3.2, the curve classes are also linearly generated. By induction assume that for all $m<n$ and all $k<m$, the effective cone of $k$ cycles 
of the blowup of $\mathbb{P}^{m}$ in $m+2$ linearly general points is linearly generated. We carry out the inductive step for $\mathbb{P}^{n}$. Let $Y, L$ be as above. By Lemma 3.3, we may assume that $Y$ is not contained in $L$. If $b_{n+1}+b_{n+2} \leq a$, then we already proved that the class of $Y$ is linearly generated. If $b_{n+1}+b_{n+2}>a$, then, by Lemma 2.10, $Y$ contains the line $l_{n+1, n+2}$ spanned by $p_{n+1}$ and $p_{n+2}$ with multiplicity at least $b_{n+1}+b_{n+2}-a$. Let $p_{0}$ denote the point of intersection $L \cap l_{n+1, n+2}$. Then the proper transform of $L \cap Y$ is an effective cycle in the blowup of $L$ in $p_{0}, p_{1}, \ldots, p_{n}$ with class

$$
a H_{L, k-1}-\left(b_{n+1}+b_{n+2}-a+c\right) E_{L, 0, k-1}-\sum_{i=1}^{n} b_{i} E_{L, i, k-1},
$$

where $c \geq 0$. By induction on $n$, this class is linearly generated. Hence

$$
k a \geq b_{n+1}+b_{n+2}-a+c+\sum_{i=1}^{n} b_{i}, \quad \text { therefore } \quad(k+1) a \geq \sum_{i=1}^{n+2} b_{i} .
$$

By Lemma 2.6, the class of $Y$ is linearly generated. This concludes the proof.

Example 3.4. Lemma 3.2 is sharp in the sense that there exist sets $\Gamma$ of $r>2 n$ points in general linear position such that $\overline{\mathrm{Eff}}_{1}\left(X_{\Gamma}^{n}\right)$ is not linearly generated. For example, let $\Gamma$ be $r>2 n$ points on a rational normal curve $C$ in $\mathbb{P}^{n}$. Points on a rational normal curve are in general linear position [Harris 1995]. Then the class of the proper transform of $C$ is

$$
n H_{1}-\sum_{i=1}^{r} E_{i, 1}
$$

Since $r>2 n$, this class cannot be in the span of the classes of lines. In the next section we will see that we can improve the bounds for linear generation exponentially if instead of assuming that $\Gamma$ is linearly general we assume $\Gamma$ is a set of very general points in $\mathbb{P}^{n}$.

More generally, let $Y$ be the cone over a rational normal curve of degree $n-k+1$ with vertex $V$ a $\mathbb{P}^{k-2}$. Let $\Gamma$ be the union of a set of $k-1$ general points $p_{1}, \ldots, p_{k-1}$ in $V$ and a set of $r-k+1$ general points $p_{k}, \ldots, p_{r}$ on $Y$. Then $\Gamma$ is in general linear position. The class of the proper transform of $Y$ is

$$
(n-k+1) H_{k}-\sum_{i=1}^{k-1}(n-k+1) E_{i, k}-\sum_{i=k}^{r} E_{i, k},
$$

which cannot be in the span of linear spaces if $r>2 n-k+1$. Consequently, we conclude the following. 
Proposition 3.5. There exist sets $\Gamma$ of $r>2 n-k+1$ points in general linear position in $\mathbb{P}^{n}$ such that $\overline{\operatorname{Eff}}_{k}\left(X_{\Gamma}^{n}\right)$ is not linearly generated.

In view of Proposition 3.5, it is natural to ask whether the bound in Theorem 3.1 can be improved to $r \leq 2 n-k+1$.

Question 3.6. Assume that $\Gamma$ is a set of $r$ linearly general points in $\mathbb{P}^{n}$ such that

$$
\max \left(n+2, n+\frac{n}{k}\right)<r \leq 2 n-k+1 .
$$

Is $\overline{\mathrm{Eff}}_{k}\left(X_{\Gamma}^{n}\right)$ linearly generated?

The answer is affirmative for curves and divisors. We will shortly check that for 2-cycles in $\mathbb{P}^{4}$ the answer is also affirmative. In Theorem 4.5 we will see that the answer is also affirmative if the points are very general. In view of this evidence, we expect the answer to Question 3.6 to be affirmative.

Remark 3.7. The dimension of the space $\mathcal{S}_{n-k, k+1}\left(\mathbb{P}^{n}\right)$ of scrolls of dimension $n-k$ and degree $k+1$ in $\mathbb{P}^{n}$ is

$$
2 n+2 n k-k^{2}-2
$$

[Coskun 2008, Lemma 2.4]. There are scrolls in $\mathcal{S}_{n-k, k+1}\left(\mathbb{P}^{n}\right)$ passing through $2 n-k+2$ points (see [Coskun 2006] for the surface case). Hence the family of scrolls passing through $2 n-k+1$ points covers $\mathbb{P}^{n}$. By Lemma 2.6, an affirmative answer to Question 3.6 is equivalent to the statement that every effective $k$-dimensional cycle intersects the proper transform of a scroll passing through the $2 n-k+1$ points nonnegatively.

Question 3.8. Let $\Gamma$ be $2 n-k+1$ linearly general points in $\mathbb{P}^{n}$. For every effective $k$-cycle $Y$ in $X_{\Gamma}^{n}$, does there exist a scroll $S$ of dimension $n-k$ and degree $k+1$ such that the proper transform $S$ in $X_{\Gamma}^{n}$ intersects $Y$ in finitely many points?

By Remark 3.7, an affirmative answer to Question 3.8 implies an affirmative answer to Question 3.6.

Effective 2-cycles on the blowup of $\mathbb{P}^{\mathbf{4}}$ at 7 points. We now verify that the answer to Question 3.6 is affirmative for two-cycles in $\mathbb{P}^{4}$. The argument is subtle because we need to verify linear generation for every configuration of 7 points in linear general position, rather than just very general configurations of points.

Theorem 3.9. Let $\Gamma$ be 7 linearly general points on $\mathbb{P}^{4}$. Then the cone $\overline{\operatorname{Eff}}_{2}\left(X_{\Gamma}^{4}\right)$ is linearly generated.

Proof. There is a unique rational normal quartic curve $R$ containing 7 linearly general points in $\mathbb{P}^{4}$ [Harris 1995]. The secant variety, $\operatorname{Sec}(R)$, to $R$ is a cubic hypersurface which has multiplicity two along $R$. Hence its proper transform $\overline{\operatorname{Sec}}(R)$ 
in $X_{\Gamma}^{4}$ has class $3 H-\sum_{i=1}^{7} 2 E_{i}$. In fact, this secant variety is a (-1)-divisor on $X_{\Gamma}^{4}$; it is in the Cremona orbit of one of the exceptional divisors.

Let $Y$ be an irreducible surface in $X_{\Gamma}^{4}$. Without loss of generality, we may assume that $Y$ is not contained in an exceptional divisor and has class $a H_{2}-\sum_{i=1}^{7} b_{i} E_{i, 2}$ with $a \geq b_{i} \geq 0$. First, suppose that $Y$ is not contained in $\overline{\operatorname{Sec}}(R)$. The class of a quadric $[Q]=2 H-\sum_{i=1}^{7} E_{i}$ is nef, and so by Lemma 2.9 we have

$$
Y \cdot Q \cdot \overline{\operatorname{Sec}}(R)=6 a-\sum_{i=1}^{7} 2 b_{i} \geq 0 .
$$

Lemma 2.6 implies that $[Y]$ is in the span of the classes of planes.

We are reduced to showing that if $Y \subset \overline{\operatorname{Sec}}(R)$, then $[Y]$ is in the span of the classes of planes. Let $\mathcal{S}_{3}$ denote the space of cubic surface scrolls containing the points of $\Gamma$. We will show the following.

Theorem 3.10. The proper transform $\bar{S}$ of a general member $S \in \mathcal{S}_{3}$ intersects $\overline{\operatorname{Sec}}(R)$ in an irreducible curve $B$ whose projection to $\operatorname{Sec}(R)$ is a degree 9 curve with multiplicity two at the points of $\Gamma$. Furthermore, the curve $B$ can be made to pass through a general point of $\overline{\operatorname{Sec}}(R)$.

Assume Theorem 3.10. Let $p \in \overline{\operatorname{Sec}}(R)$ be a general point not contained in $Y$. Hence an irreducible curve $B$ passing through $p$ intersects $Y$ in finitely many points. Let $\bar{S}$ be the proper transform of a scroll $S \in \mathcal{S}_{3}$ containing $p$ and intersecting $\overline{\operatorname{Sec}}(R)$ in an irreducible curve. We conclude that $\bar{S}$ and $Y$ intersect in finitely many points, hence their intersection number is nonnegative. Therefore,

$$
[\bar{S}] \cdot[Y]=\left(3 H_{2}-\sum_{i=1}^{7} E_{i, 2}\right) \cdot\left(a H_{2}-\sum_{i=1}^{7} b_{i} E_{i, 2}\right)=3 a-\sum_{i=1}^{7} b_{i} \geq 0
$$

By Lemma 2.6, we conclude that $[Y]$ is in the span of the classes of planes.

There remains to prove Theorem 3.10, which we will do via a series of claims. We first set some notation.

Notation 3.11. Let $l_{i, j}$ denote the line spanned by $p_{i}, p_{j} \in \Gamma$ and let $\Pi_{i_{1}, \ldots, i_{l}}$ denote the linear space spanned by $p_{i_{1}}, \ldots, p_{i_{l}} \in \Gamma$. Let $\Gamma_{i_{1}, \ldots, i_{l}}$ denote the set of points $p_{i_{1}}, \ldots, p_{i_{l}}$. Let $l$ be the line of intersection $\Pi_{1,2,3,4} \cap \Pi_{5,6,7}$ and, for $5 \leq i<j \leq 7$, let $z_{i, j}$ denote the point of intersection $\Pi_{1,2,3,4} \cap l_{i, j}$. Since the points are in linearly general position, the line $l$ does not intersect the lines $l_{i, j}$ for $1 \leq i<j \leq 4$ and intersects the planes $\Pi_{i, j, k}$, for $1 \leq i<j<k \leq 4$, in a unique point different from $z_{i, j}$.

Next, we recall a compactification $\overline{\mathcal{S}}_{3}$ of $\mathcal{S}_{3}$. Every irreducible cubic scroll induces a degree 3 rational curve in the Grassmannian $\mathbb{G}(1,4)$ of lines in $\mathbb{P}^{4}$. We can compactify the space of degree 3 rational curves in $\mathbb{G}(1,4)$ via the Kontsevich moduli space. Hence we can take the closure of $\mathcal{S}_{3}$ in the Kontsevich moduli space 
(see [Coskun 2006, §3] for details). More precisely, let $\overline{\mathcal{M}}_{0,7}(\mathbb{G}(1,4), 3)$ denote the Kontsevich moduli space of 7-pointed genus-0 maps of degree 3 to $\mathbb{G}(1,4)$. It is equipped with 7 evaluation morphisms ev ${ }_{i}: \overline{\mathcal{M}}_{0,7}(\mathbb{G}(1,4), 3) \rightarrow \mathbb{G}(1,4), 1 \leq i \leq 7$. Define

$$
\overline{\mathcal{S}}_{3}=\bigcap_{i=1}^{7} \mathrm{ev}_{i}^{-1}\left(\Sigma_{3}\left(p_{i}\right)\right),
$$

where $\Sigma_{3}\left(p_{i}\right)$ denotes the Schubert variety of lines containing $p_{i}$.

Claim 3.12. The space $\overline{\mathcal{S}}_{3}$ is irreducible of dimension 4.

Proof. The locus $\overline{\mathcal{T}}=\cap_{i=1}^{4} \mathrm{ev}_{i}^{-1}\left(p_{i}\right)$ in the Kontsevich moduli space $\overline{\mathcal{M}}_{0,4}\left(\mathbb{P}^{3}, 3\right)$ of 4-pointed genus 0 maps of degree 3 to $\mathbb{P}^{3}$ provides a compactification of the space of twisted cubic curves in $\Pi_{1,2,3,4}$ containing $\Gamma_{1,2,3,4}$. Since $\overline{\mathcal{M}}_{0,4}\left(\mathbb{P}^{3}, 3\right)$ is irreducible of dimension 16, every component of $\overline{\mathcal{T}}$ has dimension at least 4 .

If a twisted cubic $T$ is irreducible, then any finite set of points on $T$ is linearly general. Furthermore, given 6 linearly general points in $\mathbb{P}^{3}$, there is a unique twisted cubic curve containing them. Consider the incidence correspondence $I=\left\{\left(T, q_{1}, q_{2}\right) \mid q_{1}, q_{2} \in T\right\}$, where $T$ is a twisted cubic curve containing the set of points $\Gamma_{1,2,3,4}$ and $q_{1}, q_{2}$ are points such that $\Gamma_{1,2,3,4} \cup\left\{q_{1}, q_{2}\right\}$ are in linearly general position. The incidence correspondence $I$ is irreducible of dimension 6 since it is isomorphic to an open subset of $\mathbb{P}^{3} \times \mathbb{P}^{3}$. It dominates the space of twisted cubic curves containing $p_{1}, \ldots, p_{4}$ via the first projection. Since the fibers of the first projection are two-dimensional, we conclude that the space of irreducible twisted cubics containing $\Gamma_{1,2,3,4}$ is irreducible of dimension 4.

Since there are no connected curves of degree two or one containing 4 points in linearly general position in $\mathbb{P}^{3}$, any map in $\overline{\mathcal{T}}$ is birational to its image. If there is a reducible curve of degree 3 containing $\Gamma_{1,2,3,4}$, either a degree two curve must contain 3 of the points or a line must contain two of the points. In either case, it is easy to see that there is a 3-dimensional family of reducible cubics containing $\Gamma_{1,2,3,4}$. Hence these cannot form a component of $\overline{\mathcal{T}}$ and $\overline{\mathcal{T}}$ is irreducible.

Furthermore, 2 additional points $q_{1}$ and $q_{2}$ impose independent conditions on twisted cubics unless they are coplanar with 3 of the points in $\Gamma_{1,2,3,4}$ or 1 of the points is collinear with 2 of the points in $\Gamma_{1,2,3,4}$. If $q_{1}$ is collinear with $p_{1}$ and $p_{2}$, then there is a 1-parameter family of reducible cubics containing the line $l_{1,2}$. Similarly, if $q_{1}$ and $q_{2}$ are in $\Pi_{1,2,3}$ but no 4 of the points are collinear, then there is a 1-parameter family of reducible cubics containing the conic through $\Gamma_{1,2,3} \cup\left\{q_{1}, q_{2}\right\}$. If $q_{1}$ and $q_{2}$ are collinear with $p_{1}$ and $p_{2}$, there is a 3-parameter family of reducible cubics containing $l_{1,2}$. Recall that $l=\Pi_{1,2,3,4} \cap \Pi_{5,6,7}$. In particular, the subset of $\overline{\mathcal{T}}$ that parametrizes twisted cubics incident or secant to $l$ has dimension 3 or 2 respectively, since any pair of distinct points impose independent conditions on twisted cubics. Similarly, the locus of twisted cubics in $\overline{\mathcal{T}}$ passing through $z_{5,6}$ has dimension 2 . 
Since $\overline{\mathcal{M}}_{0,7}(\mathbb{G}(1,4), 3)$ is irreducible of dimension 25 [Coskun 2006, §2], every irreducible component of $\overline{\mathcal{S}}$ has dimension at least 4 . Let $T$ be a twisted cubic curve containing $\Gamma_{1,2,3,4}$, not secant to the line $l$, and not containing the points $z_{5,6}, z_{5,7}$ and $z_{6,7}$. Then there is a unique cubic scroll $S$ containing $T$ and passing through $p_{5}, p_{6}, p_{7}$ [Coskun 2006, Example A1]. Briefly, take a general $\mathbb{P}^{3}$ containing $\Pi_{5,6,7}$. This $\mathbb{P}^{3}$ intersects $T$ in 3 points $r_{1}, r_{2}, r_{3}$. There is a unique twisted cubic curve $T^{\prime}$ containing $r_{1}, r_{2}, r_{3}$ and $\Gamma_{5,6,7}$. The curves $T$ and $T^{\prime}$ are both isomorphic to $\mathbb{P}^{1}$ and there is a unique isomorphism $\phi$ taking $r_{i} \in T$ to $r_{i} \in T^{\prime}$. Then the surface $S_{T, T^{\prime}}$ swept out by lines joining the points that correspond under $\phi$ is the unique cubic scroll containing $T$ and $\Gamma_{5,6,7}$. If $T$ contains the point $z_{5,6}$ or is secant to the line $l$, then there is a 1-parameter family of choices for $T^{\prime}$. Once we fix $T$ and $T^{\prime}$, the scroll is uniquely determined by a similar construction. Since the locus of $T$ containing $z_{5,6}$ or secant to $l$ has codimension 2 , this locus cannot form a component of $\overline{\mathcal{S}}_{3}$. Finally, reducible cubic surfaces containing $\Gamma$ must contain a plane through 3 of the points and a quadric surface through the remaining 4 points. There is a 2-dimensional family of such surfaces and they do not give rise to a component in $\overline{\mathcal{S}}_{3}$ (see [Coskun 2006]). We conclude that $\overline{\mathcal{S}}_{3}$ is irreducible of dimension 4.

Claim 3.13. There exists a dense open set $U \subset \overline{\mathcal{S}}_{3}$ such that $S \not \subset \operatorname{Sec}(R)$ for $S \in U$. Furthermore, $S$ can be made to pass through a general point of $\operatorname{Sec}(R)$.

Proof. It suffices to exhibit one $S \in \overline{\mathcal{S}}_{3}$ such that $S \not \subset \operatorname{Sec}(R)$. Given 7 points in general linear position and 2 general additional points, [Coskun 2006, Example A1] shows that there are 2 cubic scrolls containing these nine points. In particular, if we take one of the two additional points outside $\operatorname{Sec}(R)$, we obtain a scroll not contained in $\operatorname{Sec}(R)$. Furthermore, a general twisted cubic in $\Pi_{1,2,3,4}$ containing $\Gamma_{1,2,3,4}$ intersects $\operatorname{Sec}(R)$ in a another point $q$. Consequently, the construction in the proof of Claim 3.12 exhibits a cubic scroll containing $q$ and not contained in $\operatorname{Sec}(R)$. Since the space $\overline{\mathcal{S}}_{3}$ is irreducible, the general scroll containing a general point of $\operatorname{Sec}(R)$ and $\Gamma$ will not be contained in $\operatorname{Sec}(R)$.

Claim 3.14. There exists a dense open set $U \subset \overline{\mathcal{S}}_{3}$ such that for $S \in U$ :

(1) The intersection $\bar{S} \cap \overline{\operatorname{Sec}}(R) \cap E_{i}$ is a finite set of points in $X_{\Gamma}^{4}$ for every $1 \leq i \leq 7$.

(2) The scroll $S$ does not contain any lines $l_{i, j}$ for $1 \leq i<j \leq 7$.

(3) The scroll $S$ does not contain any conics through 3 of the points in $\Gamma$.

(4) The scroll $S$ does not contain a twisted cubic curve through 5 of the points of $\Gamma$.

(5) The scroll $S$ does not contain the rational normal quartic $R$.

(6) The scroll $S$ does not contain a quintic curve double at one of the points of $\Gamma$ and passing through the others.

(7) The directrix of the scroll does not contain any of the points in $\Gamma$. 
Proof. Since each of these conditions are closed conditions and $\overline{\mathcal{S}}_{3}$ is irreducible, it suffices to exhibit one element $S \in \overline{\mathcal{S}}_{3}$ satisfying each condition. For (1), there exists a twisted cubic containing $\Gamma_{1,2,3,4}$ with any tangent line at $p_{1}$ (for example, the reducible twisted cubic consisting of any line through $p_{1}$ and a conic through $\Gamma_{2,3,4}$ ). Hence the tangent spaces to the scrolls at $p_{1}$ sweep out $E_{1}$ and there exists $S$ such that $\bar{S} \cap E_{1} \not \subset \overline{\operatorname{Sec}}(R)$. By permuting indices, we conclude (1).

For (2) and (3), take the scroll $S_{T, T^{\prime}}$ constructed in the proof of Claim 3.12. Since $\Pi_{1,2,3,4} \cap S_{T, T^{\prime}}=T$, this scroll does not contain any of the linear $l_{i, j}$ with $1 \leq i<j \leq 4$ or any conic passing through any of the three points in $\Gamma_{1,2,3,4}$. By permuting indices, we conclude (2) and (3).

Since a twisted cubic curve spans a $\mathbb{P}^{3}$ and the points are in linearly general position (4) is clear. For (5), (6) and (7), it is more convenient to exhibit a reducible scroll satisfying these properties. Let $S$ be the union of the plane $\Pi_{5,6,7}$ and a general quadric surface $Q$ containing $l$ and $\Gamma_{1,2,3,4}$. After choosing a point of $l$, this surface determines a point $p$ of $\overline{\mathcal{S}}_{3}$ [Coskun 2006]. The directrix line is then the unique line on the quadric $Q$ intersecting $l$ at $p$. Hence (7) holds. Since $R$ is irreducible and nondegenerate, it cannot be contained in this surface. Suppose there is a quintic curve $F$ in $S$ containing $\Gamma$ and double at $p_{1}$. Since $p_{5}, p_{6}, p_{7}$ are not collinear, $F$ must intersect $\Pi_{5,6,7}$ in a curve of degree at least 2 . Hence $F$ intersects $Q$ in a curve of degree 3 containing $\Gamma_{2,3,4}$ and double at $p_{1}$. Any cubic double at $p_{1}$ must contain the line of ruling through $p_{1}$. Since $\Gamma_{1,2,3,4}$ are linearly general there cannot be a degree 2 curve through these points on $Q$. After permuting indices, we conclude (6) holds.

Claim 3.15. There exists a dense open set $U \subset \overline{\mathcal{S}}_{3}$ such that for $S \in U$ the intersection $S \cap \operatorname{Sec}(R)$ is an irreducible degree 9 curve double along $\Gamma$.

Proof. By Claims 3.13 and 3.14, we can find a scroll $S \not \subset \operatorname{Sec}(R)$ satisfying the conclusions of Claim 3.14. The intersection $S \cap \operatorname{Sec}(R)$ is a curve $B$ of degree 9 double along $\Gamma$. We need to show that $B$ is irreducible. Recall that a smooth cubic scroll is isomorphic to the blowup of $\mathbb{P}^{2}$ at a point. Its Picard group is generated by the directrix $e$ (the curve of self-intersection -1 ) and the class of a fiber line $f$. The intersection numbers are

$$
e^{2}=-1, \quad e \cdot f=1, \quad f^{2}=0 .
$$

The effective cone is spanned by $e$ and $f$. The canonical class is $-2 e-3 f$ and the class of $B$ is $3 e+6 f$. The degree of a curve $k e+m f$ is $k+m$. If $k>m$, then any representative contains $e$ with multiplicity $k-m$. By adjunction, the arithmetic genus of a curve in the classes $e+m f, 2 e+m f$, and $3 e+m f$ are $0, m-2$, and $2 m-5$, respectively.

It is now straightforward, but somewhat tedious, to check that $B$ cannot be 


\begin{tabular}{|c|c|c|c|}
\hline class & $\begin{array}{c}\text { the number of } \\
\text { double points of } \Gamma\end{array}$ & $\begin{array}{c}\text { the number of remaining } \\
\text { points of } \Gamma \text {, respectively }\end{array}$ & reason \\
\hline$e$ & 0 & 0 & Claim 3.14 (7) \\
$e+f$ & 0 & 2 & Claim 3.14 (3,2) \\
$e+2 f$ & 0 or 1 & 4 or 1 & Claim 3.14 (4,3,2) \\
$e+3 f$ & 0,1 or 2 & 6,3 or 0 & Claim $3.14(7,5,4,3,2)$ \\
$e+4 f$ & 0,1 or 2 & 7,5 or 2 & Claim $3.14(7,5,4,3,2)$ \\
\hline
\end{tabular}

Table 1. Possible curves with class $e+m f$.

reducible. Indeed, suppose that $B$ is reducible. Write $B=B_{1} \cup B_{2}$, where the class of $B_{1}$ is $k e+m f$ with $2 \leq k \leq 3$ and assume that $B_{1}$ does not contain any fibers as components. Furthermore, if $k=3$, we may assume that $B_{1}$ is irreducible. Otherwise, we can regroup a component with class $e+m^{\prime} f$ with $B_{2}$. Then the class of $B_{2}$ is $(3-k) e+(6-m) f$ and every fiber component of $B$ is included in $B_{2}$. By Claim 3.14 (2), a curve with class $m f$ can be double at most in $0 \leq d \leq m / 2$ points of $\Gamma$ in which case it can contain at most $m-2 d$ of the remaining points of $\Gamma$. We tabulate the possibilities for curves with class $e+m f$, see Table 1 .

First, suppose $B_{1}$ has class $3 e+m f$. By assumption, it is irreducible and by arithmetic genus considerations can have at most $2 m-5$ nodes. On the other hand, $B_{2}$ can pass through at most $6-m$ of the points. We have $2 m-5+6-m=m+1<7$ if $m<6$. Hence such a curve cannot be double at all the points of $\Gamma$.

We may therefore assume that the class of $B_{1}$ is $2 e+m f$ and the class of $B_{2}$ is $e+(6-m) f$. If $B_{1}$ is reducible, then it can have at most 2 components with classes $e+m_{1} f$ and $e+m_{2} f$. An inspection of Table 1 shows that it is not possible to make $B$ double at all points of $\Gamma$. If $B_{1}$ is irreducible, then $m \geq 2$ and its arithmetic genus is $m-2$. Hence the maximal number of double points on $B_{1}$ is $m-2$. If $m=2$, then $B_{1}$ can contain at most 6 of the points of $\Gamma$ by Claim 3.14 (5) and it is smooth at those points. Hence $B$ cannot be made double at all points of $\Gamma$ by the last line of the table. If $m=3$ and $B_{1}$ has a double point, then by Claim 3.14 (6) $B_{1}$ contains at most 5 other points of $\Gamma$. By the second to last row of the table, $B$ cannot be double at all points of $\Gamma$. If $m \geq 4$, an easy inspection of the first three rows of the table show that $B$ can have at most 6 double points. Hence $B$ is irreducible.

This concludes the proof of Theorem 3.10 and consequently of Theorem 3.9.

\section{Nonlinearly generated cones}

Recall that $X_{r}^{n}$ denotes the blowup of $\mathbb{P}^{n}$ in $r$ very general points. In this section, we study the cones of effective cycles on $X_{r}^{n}$. Our first result completely characterizes when the cone of curves is linearly generated. 
Proposition 4.1. The cone $\overline{\mathrm{Eff}}_{1}\left(X_{r}^{n}\right)$ is linearly generated if and only if $r \leq 2^{n}$.

Proof. We first observe that the linear system of quadrics through $2^{n}$ very general points is nef. Choose $n$ general quadrics $Q_{1}, \ldots, Q_{n}$ in $\mathbb{P}^{n}$. By Bertini's theorem, the intersection of these quadrics is a set of $2^{n}$ points in $\mathbb{P}^{n}$. Let $X_{0}$ be the blowup of $\mathbb{P}^{n}$ at these points. We claim that $D=2 H-\sum_{i=1}^{2^{n}} E_{i}$ is nef on $X_{0}$. Note that the proper transforms of $Q_{i}$ have class $D$ and $D$ has positive degree on curves contained in exceptional divisors $E_{i}$. Since the intersection $Q_{1} \cap \cdots \cap Q_{n}$ is finite, if $B$ is a curve on $X_{0}$ not contained in an exceptional divisor, there is a quadric $Q_{i}$ whose proper transform does not contain $B$. Consequently, $D \cdot B \geq 0$ and $D$ is nef. By [Lazarsfeld 2004, Proposition 1.4.14], $2 H-\sum_{i=1}^{2^{n}} E_{i}$ is nef for very general configurations of $2^{n}$ points as well. We conclude that if $r \leq 2^{n}$, an effective curve class in $X_{r}^{n}$ satisfies the inequalities in the assumptions of Lemma 2.6, and so every curve class is a linear combination of classes of lines.

The top self-intersection of the class $Q=2 H-\sum_{i=1}^{r} E_{i}$ on $X_{r}^{n}$ is given by $2^{n}-r$. Hence if $r>2^{n}$, the top self-intersection of $Q$ is negative and $Q$ cannot be nef by Kleiman's Theorem [Lazarsfeld 2004, Theorem 1.4.9]. Suppose the class of every effective curve is in the span of the classes of lines. The cone generated by the classes of lines is a closed cone. Hence the effective and the pseudoeffective cones coincide. Since every line has nonnegative intersection with $Q$, we conclude that $Q$ is nef. This contradiction shows that there must exist effective curves whose classes are not spanned by the classes of lines.

Corollary 4.2. If $r \geq 2^{n-k+1}+k$, then $\overline{\operatorname{Eff}}_{k}\left(X_{r}^{n}\right)$ is not linearly generated.

Proof. Let $\Gamma$ be a set of $r$ very general points. Project $\Gamma$ from the first $k-1$ points $p_{1}, \ldots, p_{k-1}$ and let $\Gamma^{\prime}$ be the set of points in $\mathbb{P}^{n-k+1}$ consisting of the images of the remaining points. Then $\Gamma^{\prime}$ is a set of $r-k+1$ very general points in $\mathbb{P}^{n-k+1}$. If $r-k+1>2^{n-k+1}$, the cone $\overline{\operatorname{Eff}}_{1}\left(X_{r-k+1}^{n-k+1}\right)$ is not linearly generated by Proposition 4.1. Fix a 1-cycle $B$ with class $a H_{1}-\sum_{i=k}^{r} b_{i} E_{i, 1}$ that is not in the span of linear spaces. In particular, $2 a<\sum_{i=k}^{r} b_{i}$. Then the class

$$
a H_{k}-\sum_{i=1}^{k-1} a E_{i, k}-\sum_{i=k}^{r} b_{i} E_{i, k}
$$

is represented in $X_{r}^{n}$ by the proper transform of the cone over $B$ with vertex the span of $p_{1}, \ldots, p_{k-1}$. The resulting $k$-cycle is not in the span of $k$-dimensional linear spaces since $(k+1) a<(k-1) a+\sum_{i=k}^{r} b_{i}$.

Question 4.3. If $r<2^{n-k+1}+k$, is $\overline{\operatorname{Eff}}_{k}\left(X_{r}^{n}\right)$ linearly generated?

Remark 4.4. The answer to Question 4.3 is affirmative for curves and divisors. For cycles of intermediate dimension, we do not know any examples with $r=$ $2^{n-k+1}+k-1$ where the cone is linearly generated. 
There has been a great deal of interest in the construction of cycles that are nef but not pseudoeffective. Such cycles were constructed on abelian varieties in [Debarre et al. 2011], and on hyperkähler varieties in [Ottem 2015]. If Question 4.3 has an affirmative answer, this would give many examples of nef classes that are not pseudoeffective. For example, if $\overline{\mathrm{Eff}}_{3}\left(X_{r}^{6}\right)$ is linearly generated for $16<r<19$, then the class $4 H_{3}-\sum_{i=1}^{r} E_{i, 3}$ would be nef but not pseudoeffective; indeed, the self-intersection of this class is negative.

We can, however, give a linear bound.

Theorem 4.5. The cone $\overline{\operatorname{Eff}}_{k}\left(X_{r}^{n}\right)$ is linearly generated if $r \leq 2 n-k+1$.

Proof. The theorem is true for $k=1$ by Proposition 4.1 and for divisors by Theorem 3.1. We will prove the general case by induction on $n$. Assume that the theorem is true for $\overline{\operatorname{Eff}}_{k}\left(X_{r}^{m}\right)$ for $r \leq 2 m-k+1$ and all $k<m<n$. Let $\Gamma$ be a set of $r$ points such that $\Gamma$ consists of $r-2$ very general points $p_{1}, \ldots, p_{r-2}$ in a hyperplane $L=\mathbb{P}^{n-1}$ and two very general points $p_{r-1}, p_{r}$ not contained in $L$. Let $L^{\prime}$ denote the proper transform of $L$ in $X_{\Gamma}^{n}$. Note that $L^{\prime} \cong X_{r-2}^{n-1}$. Let $Y$ be an irreducible $k$-dimensional subvariety of $X_{\Gamma}^{n}$ not contained in an exceptional divisor with class $a H_{k}+\sum_{i=1}^{r} b_{i} E_{i}$ on $X_{\Gamma}^{n}$. If $Y$ is contained in $L^{\prime}$, then $Y \subset X_{r-2}^{n-1}$. Since $r-2 \leq 2(n-1)-k+1$, by the induction hypothesis the class of $Y$ is linearly generated and $(k+1) a \geq \sum_{i=1}^{r} b_{i}$. If $Y$ is not contained in $L^{\prime}$, then $Z=Y \cap L^{\prime}$ is an effective cycle of dimension $k-1$. Let $p_{0}$ denote the intersection of the line $l_{1,2}$ spanned by $p_{r-1}$ and $p_{r}$ with $L$. Let $\beta=\max \left(0, b_{r-1}+b_{r}-a\right)$. Consider the blowup $X_{r-1}^{n-1}$ of $L$ along $p_{0}, p_{1}, \ldots, p_{r-2}$. Then the proper transform of $Z$ is an effective cycle in $X_{r-1}^{n-1}$ with class

$$
a H_{k-1}-(\beta+c) E_{0, k-1}-\sum_{i=1}^{r-2} b_{i} E_{i, k-1},
$$

for some $c \geq 0$. Since $r \leq 2 n-k+1$, the inductive hypothesis $r-1 \leq 2(n-1)-$ $(k-1)+1$ is satisfied. We conclude that this class is linearly generated. Consequently,

$$
k a \geq \beta+\sum_{i=1}^{r-2} b_{i} \quad \text { and hence } \quad(k+1) a \geq \sum_{i=1}^{r} b_{i} .
$$

By Lemma 2.6, the class of $Y$ is linearly generated. By Corollary 2.5, $\overline{\operatorname{Eff}}_{k}\left(X_{r}^{n}\right) \subset$ $\overline{\operatorname{Eff}}_{k}\left(X_{\Gamma}^{n}\right)$ and $\overline{\operatorname{Eff}}_{k}\left(X_{r}^{n}\right)$ is linearly generated. This concludes the induction and the proof of the theorem.

The cone $\overline{\mathbf{E f f}}_{\mathbf{2}}\left(X_{\mathbf{8}}^{\mathbf{4}}\right)$. In this subsection we prove that $\overline{\operatorname{Eff}}_{2}\left(X_{8}^{4}\right)$ is linearly generated.

Theorem 4.6. The cone $\overline{\mathrm{Eff}}_{2}\left(X_{8}^{4}\right)$ is linearly generated. 
The fact that the points are now very general means that we are in position to apply degeneration arguments. These sorts of arguments work because of the semicontinuity of multiplicities in families; a surface $S$ on the very general $X_{8}^{4}$ violating the inequality $3 a \geq \sum b_{i}$ will specialize to an effective 2-cycle with the same property (Lemma 2.4).

To illustrate the range of applicable techniques we give two different degeneration arguments to prove Theorem 4.6.

Proof 1 . Let $\Gamma$ be a configuration of 8 points in $\mathbb{P}^{4}$ such that $p_{1}, \ldots, p_{7}$ are very general points and $p_{8}$ is a general point on $\operatorname{Sec}(R)$, where $\operatorname{Sec}(R)$ is the secant variety of the rational normal curve $R$ through the points $p_{1}, \ldots, p_{7}$. Let $Y$ be an irreducible surface in $X_{\Gamma}$. If $Y$ is not contained in the strict transform $\overline{\operatorname{Sec}}(R)$, then $Y \cap \overline{\operatorname{Sec}}(R)$ is a curve and the class $3 a H_{1}-\sum_{i=1}^{7} 2 b_{i} E_{i, 1}-b_{8} E_{8,1}$ is effective. We claim that the linear system $Q=2 H-\sum_{i=1}^{7} E_{i}-2 E_{8}$ is nef on $\overline{\operatorname{Sec}}(R)$. Granting this, $Q \cdot \overline{\operatorname{Sec}(R)} \cdot Y=6 a-2 \sum_{i=1}^{8} b_{i} \geq 0$, and so $Y$ is in the span of planes. We may therefore assume that $Y \subset \overline{\operatorname{Sec}}(R)$.

To prove that $Q$ is nef on $\overline{\operatorname{Sec}}(R)$ it suffices to show that the linear system of quadrics double at $p_{8}$ and passing through $p_{1}, \ldots, p_{7}$ in $\mathbb{P}^{4}$ has base locus consisting of 8 lines, none of which are contained in $\operatorname{Sec}(R)$. Then $Q$ restricts to a semiample, and in particular, nef class on $\overline{\operatorname{Sec}}(R)$. Since $p_{8}$ is general, the only line through $p_{8}$ incident to $R$ and contained in $\operatorname{Sec}(R)$ is the unique secant line $l$ to $R$ through $p_{8}$. Suppose there was another line $p_{8} \in l^{\prime} \subset \operatorname{Sec}(R)$ incident to $R$, then the plane spanned by $l$ and $l^{\prime}$ would intersect $\operatorname{Sec}(R)$ in a completely reducible cubic curve singular along the three points of intersection with $R$ and at $p_{8}$. This is clearly impossible.

Since $p_{8}$ is general, we may assume that the secant line $l$ does not contain any of the points $p_{1}, \ldots, p_{7}$. Any effective member of the linear system $Q$ is a quadric cone with vertex at $p_{8}$. Let $q_{1}, \ldots, q_{7}$ denote the projection of $p_{1}, \ldots, p_{7}$ through $p_{8}$. These are very general 7 points in $\mathbb{P}^{3}$. The base locus of the linear system of quadrics passing through $q_{1}, \ldots, q_{7}$ in $\mathbb{P}^{3}$ is 8 points $q, q_{1}, \ldots, q_{7}$, contained in the smooth locus of the projection $R^{\prime}$ of $R$. (The curve $R^{\prime}$ is a complete intersection of 2 quadrics. There is a three-dimensional linear system of quadrics passing through $q_{1}, \ldots, q_{7}$. Any quadric in this linear system not containing $R^{\prime}$ intersects $R^{\prime}$ at a further point $q$ in the smooth locus of $R^{\prime}$.) By taking cones over these quadrics with vertex $p_{8}$, we see that the base locus of $Q$ in $\mathbb{P}^{4}$ are the 8 lines spanned by $p_{8}$ and one of $q, q_{1}, \ldots, q_{7}$. Since these are lines through $p_{8}$ incident to $R$ and distinct from the secant line containing $p_{8}$, none of them are contained in $\operatorname{Sec}(R)$.

To prove the theorem we need to show that $S \cdot Y=3 a-\sum_{i=1}^{8} b_{i} \geq 0$ for a cubic scroll $S$. It suffices to show that there is some scroll $S$ which intersects $Y$ in finitely many points. We may further assume that $Y \subset \overline{\operatorname{Sec}}(R)$. 
Let us consider the family of cubic scrolls through these 8 points. Through the first 7 points, $p_{1}, \ldots, p_{7}$ there is a 4-dimensional space of scrolls. There is an open set of this parameter space parametrizing scrolls $S$ such that the intersection $\operatorname{Sec}(R) \cap S=B$ is an irreducible curve. There exists such a curve through a general eighth point, by Claim 3.13, and so there must in fact be an irreducible curve through a general eighth point. In all, picking $p_{8}$ general, we have that the space of cubic scrolls containing $p_{1}, \ldots, p_{8}$ is of dimension 2 and there is a nonempty open subset of scrolls such that the proper transform of a scroll $\bar{S}$ intersects $\operatorname{Sec}(R)$ in an irreducible curve.

Now, taking a general point $q$ of $\operatorname{Sec}(R)$, there exists two scrolls containing the 9 points $q, p_{1}, \ldots, p_{8}$ [Coskun 2006]. Since varying $q$ gives a 2-dimensional family of scrolls, we conclude that by choosing $S$ generic, the curve $B$ can be made to pass through a general ninth point of $\operatorname{Sec}(R)$. In particular, we can choose a scroll so that $B$ is not contained in $Y$. As the curve $B$ is irreducible and not contained in $Y$, we see that $\bar{S}$ and $Y$ generically intersect in finitely many points and so their intersection number is nonnegative. We conclude that the effective cone is linearly generated.

Proof 2. We will degenerate to a configuration where the 8 points lie on two rank 3 quadrics.

Let $x_{0}, \ldots, x_{4}$ be coordinates on $\mathbb{P}^{4}$ and let $p_{1}=(1,0,0,0,0), \ldots, p_{5}=$ $(0,0,0,0,1)$ denote the coordinate points. Consider the two quadrics $q_{1}=\left\{x_{0} x_{1}+\right.$ $\left.x_{0} x_{2}+x_{1} x_{2}=0\right\}$ and $q_{2}=\left\{x_{2} x_{3}+x_{2} x_{4}+x_{3} x_{4}=0\right\}$. Here $q_{1}$ is a cone over a smooth conic in the $x_{0} x_{1} x_{2}$-plane with the vertex being the line $\left\{x_{0}=x_{1}=x_{2}=0\right\}$, and similarly for $q_{2}$. Note that $q_{1}$ and $q_{2}$ both contain the points $p_{1}, \ldots, p_{5}$. Moreover, $q_{1}$ and $q_{2}$ respectively contain $p_{4}, p_{5}$ and $p_{1}, p_{2}$ with multiplicity two. We now choose the remaining three points $p_{6}, p_{7}, p_{8}$ to be general on the intersection $q_{1} \cap q_{2}$.

On the blowup at these points the strict transform of $q_{1}$ is an irreducible divisor $Q_{1}$ with class $2 H-\sum_{i=1}^{8} E_{i}-E_{4}-E_{5}$. Consider the divisor

$$
D_{1}=3 H-2 \sum_{i=1}^{8} E_{i}+E_{4}+E_{5}
$$

it satisfies $\left[Q_{1}\right] \cdot\left[D_{1}\right]=2\left(3 H^{2}-\sum_{i=1}^{8} E_{i, 2}\right)$. A computation gives that the linear system $\left|D_{1}\right|$ is 2-dimensional. Moreover, the base locus of $D_{1}$ is 1-dimensional and has 18 components: 15 lines and 3 quartic normal curves. One checks that none of these curves lie on $Q_{1}$. Indeed, these statements are easy to verify for one particular configuration (and thus it follows a general 8-tuple as above). In particular, $\left.D_{1}\right|_{Q_{1}}$ has only finitely many base-points, and hence is nef on $Q_{1}$.

Now, suppose that $Y \subset X$ is an irreducible surface with class $a H^{2}-\sum_{i=1}^{8} b_{i} E_{i, 2}$. Then, if $Y$ is not contained in $Q_{1}$, the intersection $i^{*} Y$ is represented by an effective 
1-cycle on $Q_{1}$ (here $i: Q_{1} \rightarrow X$ is the inclusion). As $\left.D_{1}\right|_{Q_{1}}$ is nef, we have

$$
0 \leq\left. D_{1}\right|_{Q_{1}} \cdot i^{*} Y=6 a-\sum_{i=1}^{8} 2 b_{i},
$$

as desired. A symmetric argument (with $Q_{2}=2 H-\sum_{i=1}^{8} E_{i}-E_{1}-E_{2}$ and $D_{2}=3 H-2 \sum_{i=1}^{8} E_{i}+E_{1}+E_{2}$ ), shows that the conclusion also holds if $Y$ is not contained in $Q_{2}$.

We therefore reduce to the case where $Y \subseteq Q_{1} \cap Q_{2}$. Note that $Q_{1} \cap Q_{2}$ is an irreducible surface, so $Y=Q_{1} \cap Q_{2}$. Now

$$
\left[Q_{1}\right]\left[Q_{2}\right]=4 H^{2}-2 E_{1,2}-2 E_{2,2}-2 E_{3,2}-2 E_{4,2}-E_{5,2}-E_{6,2}-E_{7,2}-E_{8,2},
$$

which is equivalent to a sum of 4 planes. This completes the proof.

We immediately deduce the following corollary.

Corollary 4.7. If $X_{r}^{n}$ is a Mori dream space, then $\overline{\operatorname{Eff}}_{k}\left(X_{r}^{n}\right)$ is finitely generated.

Remark 4.8. Combining Theorem 4.6 with the degeneration argument of Theorem 4.5, it follows that $\overline{\operatorname{Eff}}_{2}\left(X_{r}^{n}\right)$ is linearly generated for $r \leq 2 n$ as long as $n \geq 4$.

We will see in the next section that $\overline{\operatorname{Eff}}_{2}\left(X_{10}^{4}\right)$ is not finitely generated, assuming the SHGH conjecture holds for blowups of $\mathbb{P}^{2}$ at 10 points. The only remaining case in dimension 4 is

Question 4.9. Is the cone $\overline{\mathrm{Eff}}_{2}\left(X_{9}^{4}\right)$ linearly generated?

It is not easy to find explicit curves in $X_{r}^{n}$ which are not in the span of lines. The following example gives a construction in the case of 9 very general points in $\mathbb{P}^{3}$. Example 4.10. The class $C_{C M}=57 H_{1}-\sum_{i=1}^{10} 18 E_{i, 1}$ on $X_{10}^{2}$ is represented by a unique irreducible plane curve of genus 10, by [Ciliberto and Miranda 2011]. On $X_{9}^{3}$ there is a unique divisor $Q$ in the class $2 H_{1}-\sum_{i=1}^{9} E_{i, 1}$, given by the strict transform of the unique quadric through the 9 points. There is a morphism $i: X_{10}^{2} \rightarrow X_{9}^{3}$ identifying the proper transform of $Q$ with the blowup of $\mathbb{P}^{2}$ at 10 points.

A quick calculation shows that the pushforward of the class of $C_{C M}$ to $X_{9}^{3}$ is

$$
i_{*}\left(C_{C M}\right)=78 H_{1}-21 E_{1,1}-\sum_{i=2}^{9} 18 E_{i, 1} .
$$

We have $21+8(18)=165$, while $2 \cdot 78=156$. Hence this curve is not in the span of the lines. It does not, however, define an extremal ray on $\overline{\mathrm{Eff}}_{1}\left(X_{9}^{3}\right)$. In the next section we will use a similar construction to show that, assuming the SHGH conjecture, the cone $\overline{\operatorname{Eff}}_{1}\left(X_{9}^{3}\right)$ is not finitely generated.

By repeatedly taking cones over $i_{*}\left(C_{C M}\right)$, we obtain explicit nonlinearly generated codimension-two cycles on $X_{n+6}^{n}$ for every $n \geq 3$. 
Complete intersections also provide examples of nonlinearly generated pseudoeffective curve classes, provided that the number of points is large.

Example 4.11. Assume that $d^{n} \geq r>2 d^{n-1}$ for some integer $d>2$. Then the divisor class $D=d H-\sum_{i=1}^{r} E_{i}$ is nef on $X_{r}^{n}$ by the argument given in the proof of Proposition 4.1. The $(n-1)$-fold self-intersection of the class is

$$
D^{n-1}=d^{n-1} H_{1}-\sum_{i=1}^{r} E_{i, 1} .
$$

Since $r>2 d^{n-1}$, this class is not in the span of lines. On the other hand, the class is pseudoeffective. A small perturbation of $D$ is ample. Hence a sufficiently high multiple is very ample and the (n-1)-fold self-intersection is an effective curve. It follows that the class $D^{n-1}$ is pseudoeffective.

\section{Nonfinitely generated cones}

The cone of curves of the blowup of $\mathbb{P}^{2}$ at 10 or more very general points is not entirely understood, and we will find it useful to assume the following standard conjecture.

Conjecture 5.1 (Segre-Harbourne-Gimigliano-Hirschowitz (SHGH) conjecture [Gimigliano 1987]). Suppose that $r \geq 10$ and that $m_{1} \geq m_{2} \geq \cdots \geq m_{r}$ and $d>m_{1}+m_{2}+m_{3}$. Then

$$
H^{0}\left(X_{r}^{2}, d H_{1}-\sum_{i=1}^{r} m_{i} E_{i, 1}\right)=\left(\begin{array}{c}
d+2 \\
2
\end{array}\right)-\sum_{i=1}^{r}\left(\begin{array}{c}
m_{i}+1 \\
2
\end{array}\right) .
$$

We next prove that the cone of codimension-2 cycles on $X_{r}^{n}$ is not finitely generated for $r \geq n+6$, assuming the SHGH conjecture. The calculation relies on the following observation of de Fernex.

Theorem 5.2 [de Fernex 2010, Proposition 3.4]. Assume the SHGH conjecture holds for 10 points. Let $P \subset N^{1}\left(X_{10}^{2}\right)$ be the positive cone

$$
P=\left\{D \in N^{1}\left(X_{10}^{2}\right): D^{2} \geq 0, D \cdot H \geq 0\right\},
$$

where $H$ is an ample divisor. Then

$$
\overline{\operatorname{Eff}}_{1}\left(X_{10}^{2}\right) \cap K_{\geq 0}=P \cap K_{\geq 0} .
$$

Let $Q \subset X_{9}^{3}$ be the strict transform of the unique quadric passing through the 9 points and let $i$ denote the inclusion of $Q$ in $X_{9}^{3}$. Note that $Q$ is isomorphic to $X_{10}^{2}$, and so Conjecture 5.1 provides some information about the cone $\overline{\operatorname{Eff}}_{1}(Q)$. However, the map $N_{1}(Q) \rightarrow N_{1}\left(X_{9}^{3}\right)$ is not injective, since the two rulings of the quadric both 
map to the class of a line in $\mathbb{P}^{3}$. The next lemma gives a criterion to show that certain extremal rays on $\overline{\operatorname{Eff}}_{1}(Q)$ nevertheless push forward to extremal rays on $\overline{\operatorname{Eff}}_{1}\left(X_{9}^{3}\right)$.

Write $r_{1}$ and $r_{2}$ for the classes of the two rulings on the quadric, and let $f_{i}=\left.E_{i}\right|_{Q}$ be the exceptional curves. Let $\ell_{i j}=r_{1}-f_{i}-f_{j} \in N_{1}(Q)$, this class is not effective on $Q$, but $i_{*} \ell_{i j}$ is effective in $N_{1}\left(X_{9}^{3}\right)$ since it is the class of a line through the points $p_{i}$ and $p_{j}$.

Theorem 5.3. Suppose that $D$ is a class in $N_{1}(Q)$ which satisfies:

(1) $D$ is nef, and if $\gamma \in \overline{\operatorname{Eff}}_{1}(Q)$ has $D \cdot \gamma=0$, then $\gamma$ is a multiple of $D$.

(2) $D \cdot r_{1}=D \cdot r_{2}$.

(3) $D \cdot \ell_{i j}>0$ for all $i$ and $j$.

Then $i_{*} D$ is nonzero and spans an extremal ray on $\overline{\operatorname{Eff}}_{1}\left(X_{9}^{3}\right)$.

Proof. That $i_{*} D$ is nonzero follows from the fact that $D$ is nef, since if $H$ is ample then so is $i^{*} H$ and then $i_{*} D \cdot H=D \cdot i^{*} H>0$. We claim next that $D$ lies on a two-dimensional extremal face of the cone

$$
\Sigma=\overline{\operatorname{Eff}}_{1}(Q)+\sum_{i, j} \mathbb{R}_{\geq 0}\left[\ell_{i j}\right]+\mathbb{R}\left[r_{1}-r_{2}\right] \subset N_{1}(Q) .
$$

More precisely, if $D=\alpha+\beta$ with $\alpha, \beta \in \Sigma$, then

$$
\alpha=a_{1} D+b_{1}\left(r_{1}-r_{2}\right) \quad \text { and } \beta=a_{2} D+b_{2}\left(r_{1}-r_{2}\right),
$$

where $a_{1}$ and $a_{2}$ are positive. Note that $D$ is nef, $D \cdot\left(r_{1}-r_{2}\right)=0, D \cdot \ell_{i j}>0$ for all $i, j$, and $D \cdot f_{k}>0$. Hence $D$ is contained in the dual cone of $\Sigma$. By conditions (1) and (2), the classes in $\Sigma$ with $D \cdot C=0$ are precisely $\mathbb{R}_{\geq 0} D+\mathbb{R}\left(r_{1}-r_{2}\right)$.

We claim next that

$$
\overline{\operatorname{Eff}}_{1}\left(X_{9}^{3}\right)=i_{*} \overline{\operatorname{Eff}}_{1}(Q)+\sum_{i, j} \mathbb{R}_{\geq 0} i_{*}\left[\ell_{i j}\right]=i_{*} \Sigma .
$$

Suppose that $\Gamma$ is an irreducible effective cycle on $\overline{\operatorname{Eff}}_{1}\left(X_{9}^{3}\right)$. If $Q \cdot \Gamma<0$, then $\Gamma$ must be contained in $Q$, and so $[\Gamma]$ is contained in $i_{*} \overline{\operatorname{Eff}}_{1}(Q)$. If $Q \cdot \Gamma \geq 0$, then $\Gamma$ satisfies $2 a \geq \sum_{i} b_{i}$, which means that $[\Gamma]$ is in the span of classes of lines $i_{*}\left[\ell_{i j}\right]$ and lines $i_{*}\left[f_{k}\right]$ in the exceptional divisors, by Lemma 2.6. Each $f_{k}$ is numerically equivalent to a curve in the quadric.

Suppose now that $i_{*} D=\alpha+\beta$, where $\alpha$ and $\beta$ are pseudoeffective classes on $\overline{\operatorname{Eff}}_{1}\left(X_{9}^{3}\right)$. Using the decomposition above, we can write $\alpha=i_{*} \alpha_{Q}+\sum c_{i j} i_{*}\left[\ell_{i j}\right]$ and $\beta=i_{*} \beta_{Q}+\sum d_{i j} i_{*}\left[\ell_{i j}\right]$, where $\alpha_{Q}$ and $\beta_{Q}$ are classes in $\overline{\operatorname{Eff}}_{1}(Q)$.

We claim now that

$$
D=\alpha_{Q}+\beta_{Q}+\sum\left(c_{i j}+d_{i j}\right) \ell_{i j}+f\left(r_{1}-r_{2}\right),
$$


for some constant $f$. Indeed, the two sides differ by an element of the kernel of $i_{*}: N_{1}(Q) \rightarrow N_{1}\left(X_{9}^{3}\right)$, which is generated by $r_{1}-r_{2}$, giving rise to the constant $f$.

Since $D^{2}=0, D \cdot\left(r_{1}-e_{i}-e_{j}\right)>0$ for any $i$ and $j$, and $D \cdot\left(r_{1}-r_{2}\right)=0$, it must be that $c_{i j}=d_{i j}=0$ for all $i$ and $j$. We conclude that

$$
\alpha_{Q}=a_{1} D+b_{1}\left(r_{1}-r_{2}\right) \text { and } \beta_{Q}=a_{2} D+b_{2}\left(r_{1}-r_{2}\right) .
$$

Hence $\alpha=i_{*} \alpha_{Q}=a_{1} i_{*} D$ and $\beta=i_{*} \beta_{Q}=a_{2} i_{*} D$. This shows that $i_{*} D$ is extremal.

The requirement that $D$ is nef makes it difficult to exhibit classes $D$ on $X_{10}^{2}$ with the necessary properties without assuming the description of $\overline{\operatorname{Eff}}_{1}\left(X_{10}^{2}\right)$ provided by the SHGH conjecture.

Theorem 5.4. Assume that the SHGH conjecture holds for blowups of $\mathbb{P}^{2}$ at 10 very general points. Then there exist infinitely many classes D satisfying the hypotheses of Theorem 5.3.

Proof. It is convenient to fix an identification $Q \cong X_{10}^{2}$ and rewrite the hypotheses in the basis for $N^{1}(Q)$ arising from this identification. The strict transforms of the two rulings through the point $p_{1}$ give disjoint $(-1)$-curves on $Q$, and these can be contracted. The other 8 exceptional curves $f_{i}$ can then be contracted to give a map to $\mathbb{P}^{2}$. Let $e_{0}$ and $e_{1}$ be the first two $(-1)$-curves contracted, and let $e_{j}=f_{j}$ for $2 \leq j \leq 8$. With respect to this new basis, we have $r_{1}-f_{1}=e_{0}$ and $r_{2}-f_{1}=e_{1}$, and $f_{1}=h-e_{0}-e_{1}$, where $h$ denotes the class of a line on $\mathbb{P}^{2}$.

While the first condition in Theorem 5.3 is independent of the basis, the last two can be rewritten as:

$$
D \cdot e_{0}=D \cdot e_{1}
$$

$$
D \cdot e_{0}>D \cdot e_{j} \text { for any } j>1 \text {, and } D \cdot\left(h-e_{1}-e_{i}-e_{j}\right)>0 \text { for any } i, j>1 \text {. }
$$

The first part of $\left(3^{\prime}\right)$ arises when $i=1<j$, while the second case is when $1<i<j$. Fix any $\frac{1}{\sqrt{10}}<\delta<\frac{1}{3}$, and let $\delta^{\prime}=\sqrt{\frac{1}{8}\left(1-2 \delta^{2}\right)}$. Observe that $\frac{3}{10}<\frac{1}{6} \sqrt{\frac{7}{2}}<\delta^{\prime}<\delta$ for $\delta$ in this range. Consider the divisor

$$
D_{\delta}=h-\delta\left(e_{0}+e_{1}\right)-\delta^{\prime} \sum_{j=2}^{9} e_{j} .
$$

We check each of the hypotheses in turn. To simplify notation, for the rest of this proof set $X=X_{10}^{2}$.

(1) First we check that $D_{\delta}$ is nef. The cone theorem implies that

$$
\overline{\operatorname{Eff}}_{1}(X)=\overline{\operatorname{Eff}}_{1}(X)_{K_{X} \geq 0}+\sum_{i} \mathbb{R}_{\geq 0}\left[C_{i}\right]
$$


where the $C_{i}$ are $K_{X}$-negative curves. According to Theorem 5.2,

$$
\overline{\operatorname{Eff}}_{1}(X) \cap \overline{\operatorname{Eff}}_{1}(X)_{K_{X} \geq 0}=P \cap K_{\geq 0} .
$$

Hence it suffices to show that $D_{\delta} \cdot C \geq 0$ if $C$ is $K_{X}$-negative, and that $D_{\delta} \cdot C \geq 0$ if $C$ has $C^{2} \geq 0$ and $C \cdot H>0$.

First, suppose that $C$ is a pseudoeffective class with $K_{X} \cdot C<0$. We have

$$
3 D_{\delta}-K_{X}=(3 \delta-1)\left(e_{0}+e_{1}\right)+\left(3 \delta^{\prime}-1\right) \sum_{j=2}^{9} e_{j},
$$

and so

$$
3 D_{\delta} \cdot C=K_{X} \cdot C+\left((3 \delta-1)\left(e_{0}+e_{1}\right)+\left(3 \delta^{\prime}-1\right) \sum_{j=2}^{9} e_{j}\right) \cdot C .
$$

However, since $\delta<\frac{1}{3}$, the number $3 \delta-1$ is negative. It is easy to check that $\delta^{\prime}<\frac{1}{3}$ as well, and so the divisor on the right is a sum of exceptional divisors with negative coefficients. If $C$ is any curve other than one of the $e_{i}$, then both terms on the right are negative. If $C$ is one of the curves $e_{i}$, then $D_{\delta} \cdot C>0$ because $\delta$ and $\delta^{\prime}$ are both positive.

It remains to check that $D_{\delta} \cdot C>0$ if $C$ is a class with positive self-intersection. This follows from the Cauchy-Schwartz inequality. Suppose that $d^{2} \geq \sum a_{i}^{2}$ and $e^{2} \geq \sum b_{i}^{2}$, then $d e \geq \sum a_{i} b_{i}$. Moreover, equality is achieved if and only if $C$ is a multiple of $D_{\delta}$.

(2') Since $D_{\delta}$ is of the form $h-\delta e_{0}-\delta e_{1}-\cdots$, we have $D \cdot e_{0}=D \cdot e_{1}$.

(3') Because $\delta>\delta^{\prime}$, we have $D \cdot e_{0}>D \cdot e_{j}$ for any $j>1$. We also have

$$
D \cdot\left(h-e_{1}-e_{i}-e_{j}\right)=1-\delta-2 \delta^{\prime}>0,
$$

since $\delta^{\prime}<\delta<\frac{1}{3}$.

Remark 5.5. One can even arrange that $D_{\delta}$ is a rational class through judicious choice of $\delta$. For example,

$$
D_{\frac{226}{692}}=h-\frac{226}{692}\left(e_{0}+e_{1}\right)-\frac{217}{692} \sum_{i=2}^{9} e_{i} .
$$

In general such classes are not expected to have any effective representatives.

Assuming the SHGH conjecture, we can now conclude that $\overline{\operatorname{Eff}}^{2}\left(X_{r}^{n}\right)$ is not finitely generated if $r \geq n+6$. We need the following lemma, which guarantees that cones over extremal classes in $\overline{\operatorname{Eff}}_{k}\left(X_{r}^{n}\right)$ are extremal in $\overline{\operatorname{Eff}}_{k+1}\left(X_{r+1}^{n+1}\right)$. 
Lemma 5.6. Suppose that $D=a H_{k}-\sum_{i=1}^{r} a_{i} E_{i, k}$ spans an extremal ray on $\overline{\operatorname{Eff}}_{k}\left(X_{r}^{n}\right)$. Then $C D=a H_{k+1}-a E_{0, k+1}-\sum_{i=1}^{r} a_{i} E_{i, k+1}$ spans an extremal ray on $\overline{\operatorname{Eff}}_{k+1}\left(X_{r+1}^{n+1}\right)$. In particular, if $\overline{\operatorname{Eff}}_{k}\left(X_{r}^{n}\right)$ has infinitely many extremal rays, then so does $\overline{\operatorname{Eff}}_{k+1}\left(X_{r+1}^{n+1}\right)$.

Lemma 5.6 immediately implies the following.

Corollary 5.7. Assume the SHGH conjecture for the blowup of $\mathbb{P}^{2}$ at 10 points. Then $\overline{\operatorname{Eff}}^{2}\left(X_{r}^{n}\right)$ is not finitely generated if $r \geq n+6$.

Proof of Lemma 5.6. Given $r+1$ very general points $p_{0}, \ldots, p_{r}$ in $\mathbb{P}^{n+1}$, their projection from $p_{0}$ gives $r$ very general points in $\mathbb{P}^{n}$. Let $D_{i}$ be effective cycles arbitrarily close to $D$ in $\overline{\operatorname{Eff}}_{k}\left(X_{r}^{n}\right)$. Then the classes of the cones $C D_{i}$ over $D_{i}$ converge to $C D$. Hence $C D \in \overline{\operatorname{Eff}}_{k+1}\left(X_{r+1}^{n+1}\right)$.

Conversely, we claim that if $C D=a H_{k+1}-a E_{0}-\sum_{i=1}^{r} b_{i} E_{i, k+1}$ is a pseudoeffective $(k+1)$-cycle on $X_{n+1}^{r+1}$, then $D=a H_{k}-\sum_{i=1}^{r} b_{i} E_{i, k}$ is a pseudoeffective $k$-cycle on $X_{n}^{r}$. The class $C D+\epsilon H_{k+1}$ is effective for any $\epsilon>0$. Let $V_{\epsilon}$ be a (rational) cycle representing the class $C D+\epsilon H_{k+1}$. Let $\ell_{0 j}$ denote the strict transform on $X_{n+1}^{r+1}$ of the line through $p_{0}$ and $p_{j}$. By Lemma 2.10, $V_{\epsilon}$ contains the line $\ell_{0 j}$ with multiplicity $\beta_{j} \geq b_{j}-\epsilon$. Let $L \subset X_{r+1}^{n+1}$ be the proper transform of a general hyperplane in $\mathbb{P}^{n+1}$. The lines $l_{0 j}$ intersect $L$ in $r$ very general points $p$. The proper transform of the intersection $L \cap V_{\epsilon}$ gives an effective cycle with class $(a+\epsilon) H_{k}-\sum_{i=1}^{j} \beta_{j} E_{j, k}$. Letting $\epsilon$ tend to 0 , we see that $a H_{k}-\sum_{i=1}^{r} b_{i} E_{i, k}$ is pseudoeffective in $X_{r}^{n}$, as required.

Now, suppose that $D=a H_{k}-\sum_{i=1}^{r} b_{i} E_{i, k}$ spans an extremal ray of $\overline{\operatorname{Eff}}_{k}\left(X_{r}^{n}\right)$. We claim that $C D=a H_{k+1}-a E_{0, k+1}-\sum_{i=1}^{r} b_{i} E_{i, k+1}$ spans an extremal ray of $\overline{\operatorname{Eff}}_{k+1}\left(X_{r+1}^{n+1}\right)$. Suppose $C D=\alpha+\beta$, where $\alpha$ and $\beta$ are both pseudoeffective $(k+1)$-cycles on $X_{r+1}^{n+1}$. Since any pseudoeffective class has $a \geq b_{0}$, it must be that $\alpha=c H_{k+1}-c E_{0, k+1}-\sum_{i=1}^{r} c_{i} E_{i, k+1} \quad$ and $\quad \beta=d H_{k+1}-d E_{0, k+1}-\sum_{i=1}^{r} d_{i} E_{i, k+1}$. Then

$$
\alpha_{0}=c H_{k}-\sum_{i=1}^{r} c_{i} E_{i, k} \quad \text { and } \quad \beta_{0}=d H_{k}-\sum_{i=1}^{r} e_{i} E_{i, k} .
$$

are pseudoeffective on $X_{r}^{n}$. Hence $\alpha_{0}$ and $\beta_{0}$ are proportional to $D$. It follows that $\alpha$ and $\beta$ are proportional to $C D$ and $C D$ is extremal.

There are several interesting remaining questions concerning the finite generation of cones of higher codimension.

Question 5.8. Can one show that $\overline{\operatorname{Eff}}_{n-2}\left(X_{r}^{n}\right)$ is not finitely generated for $r \geq n+6$ independently of the SHGH conjecture? 
Question 5.9. Fix $n$ and $k$. Does there exist $r$ for which $\overline{\operatorname{Eff}}_{k}\left(X_{r}^{n}\right)$ is not finitely generated? How does $r$ depend on $n$ and $k$ ?

In particular, we have the following fundamental question:

Question 5.10. For every $n$, does there exist $r$ for which $\overline{\operatorname{Eff}}_{1}\left(X_{r}^{n}\right)$ is not finitely generated?

If $\overline{\operatorname{Eff}}_{1}\left(X_{r}^{n}\right)$ is not finitely generated for $r \geq r_{0}$, then by Lemma 5.6 $\overline{\operatorname{Eff}}_{k}\left(X_{r+k-1}^{n+k-1}\right)$ is not finitely generated for $r \geq r_{0}$. Hence an affirmative answer to Question 5.10 implies an affirmative answer to Question 5.9.

\section{Blowups at points in special position}

Until now we have considered blowups of $\mathbb{P}^{n}$ at linearly general or very general points. It is also interesting to consider cones of effective cycles on blowups of $\mathbb{P}^{n}$ at special configurations of points. The dependence of the cones on the position of the points can be subtle, which makes degeneration arguments difficult. We will see that the property of the effective cone being finite is neither an open nor closed condition, even in families where the vector space of numerical classes of $k$-dimensional cycles has constant dimension.

Proposition 6.1. Let $\Gamma$ be a set of $r$ points whose span is $\mathbb{P}^{m} \subset \mathbb{P}^{n}$. Let $X_{\Gamma}^{n}$ and $X_{\Gamma}^{m}$ denote the blowup of $\mathbb{P}^{n}$ and $\mathbb{P}^{m}$ along $\Gamma$, respectively, then

(1) $\overline{\operatorname{Eff}}_{k}\left(X_{\Gamma}^{n}\right)$ is linearly generated for $m \leq k \leq n-1$ and

(2) $\overline{\operatorname{Eff}}_{k}\left(X_{\Gamma}^{n}\right)=\overline{\operatorname{Eff}}_{k}\left(X_{\Gamma}^{m}\right)$ for $k<m$.

Proof. Since $X_{\Gamma}^{m}$ embeds in $X_{\Gamma}^{n}$ as the proper transform of the $\mathbb{P}^{m}$ spanned by $\Gamma$, any effective cycle $Z \subset X_{\Gamma}^{m}$ is also an effective cycle in $X_{\Gamma}^{n}$ with the same class. Hence $\overline{\operatorname{Eff}}_{k}\left(X_{\Gamma}^{m}\right) \subseteq \overline{\operatorname{Eff}}_{k}\left(X_{\Gamma}^{n}\right)$ for $k<m$. Conversely, suppose that $k<m$. Let $Z$ be an effective cycle in $\mathbb{P}^{n}$ of dimension $k$ with class [Z]. We may assume that $Z$ is not contained in an exceptional divisor. Choose a general point $p$. Let $q_{i}$ denote the projection of $p_{i}$ from $p$ and let $Z^{\prime}$ be the projection of $Z$ from $p$. Then $Z^{\prime}$ and $Z$ have the same degree and the multiplicities of $Z^{\prime}$ at $q_{i}$ are greater than or equal to the multiplicities of $Z$ at $p_{i}$. Repeatedly projecting $Z$ to $\mathbb{P}^{m}$ from general points, we obtain an effective cycle contained in $\mathbb{P}^{m}$. Since $[Z]$ differs from the class of this cycle by a positive combination of exceptional linear spaces $E_{i, k}$, we conclude that $[Z]$ is effective on $X_{\Gamma}^{m}$. Taking closures, we obtain the reverse inclusion $\overline{\operatorname{Eff}}_{k}\left(X_{\Gamma}^{n}\right) \subseteq \overline{\operatorname{Eff}}_{k}\left(X_{\Gamma}^{m}\right)$.

If $k \geq m$, let $L$ be a $k$-dimensional linear space containing $\Gamma$. Then the proper transform $\bar{L}$ of $L$ has class $H_{k}-\sum_{i=1}^{r} E_{i, k}$. Since a $k$-dimensional variety not contained in an exceptional divisor has class $a H_{k}-\sum_{i=1}^{r} b_{i} E_{i, k}$, with $a \geq b_{i} \geq 0$, we conclude that any $k$-dimensional effective cycle is a nonnegative linear combination of $[\bar{L}]$ and $E_{i, k}, 1 \leq i \leq r$. 
By taking $m=1$, we obtain the following corollary.

Corollary 6.2. Suppose $\Gamma$ is a set of $r$ collinear points in $\mathbb{P}^{n}$. Then $\overline{\operatorname{Eff}}_{k}\left(X_{\Gamma}^{n}\right)$ is linearly generated for every $1 \leq k \leq n-1$.

Remark 6.3. It was shown in [Ottem 2011] that the blowup of $\mathbb{P}^{2}$ in collinear points is a Mori dream space (and indeed its Cox ring can be computed). Consequently, the cone of curves and divisors are finite polyhedral. The previous corollary generalizes this to all cycles.

Let $L \cong \mathbb{P}^{n-1}$ be a hyperplane in $\mathbb{P}^{n}$. Let $\Gamma^{\prime} \subset L$ be a set of points $p_{1}, \ldots, p_{r}$ and let $p_{0} \in \mathbb{P}^{n}$ be a point not contained in $L$. Let $\Gamma=\Gamma^{\prime} \cup\left\{p_{0}\right\}$. Let $X_{\Gamma}^{n}$ and $X_{\Gamma^{\prime}}^{n-1}$ denote the blowup of $\mathbb{P}^{n}$ and $\mathbb{P}^{n-1}$ along $\Gamma$ and $\Gamma^{\prime}$, respectively. Taking cones with vertex at $p_{0}$, we generate a subcone $\mathrm{C} \overline{\operatorname{Eff}}_{k}\left(X_{\Gamma^{\prime}}^{n-1}\right) \subset \overline{\operatorname{Eff}}_{k+1}\left(X_{\Gamma}^{n}\right)$.

Proposition 6.4. The cone $\overline{\operatorname{Eff}}_{k+1}\left(X_{\Gamma}^{n}\right)$ is generated by $\overline{\mathrm{Eff}}_{k}\left(X_{\Gamma^{\prime}}^{n-1}\right), \overline{\operatorname{Eff}}_{k+1}\left(X_{\Gamma^{\prime}}^{n-1}\right)$, and $E_{0, k+1}$. Furthermore, the extremal rays of $\mathrm{C} \overline{\mathrm{Eff}}_{k}\left(X_{\Gamma^{\prime}}^{n-1}\right)$ are also extremal in $\overline{\operatorname{Eff}}_{k+1}\left(X_{\Gamma}^{n}\right)$.

Proof. Let $Z=a H_{k+1}-\sum_{i=0}^{r} b_{i} E_{i, k+1}$ be an irreducible $(k+1)$-dimensional variety in $X_{\Gamma}^{n}$. We may assume that $Z$ is not contained in any exceptional divisors. Otherwise, its class is a positive multiple of $E_{i, k+1}$. The proper transform of $L$ in $X_{\Gamma}^{n}$ is isomorphic to $X_{\Gamma^{\prime}}^{n-1}$. If $Z$ is contained in $X_{\Gamma^{\prime}}^{n-1}$, then the class of $Z$ is in $\overline{\operatorname{Eff}}_{k+1}\left(X_{\Gamma^{\prime}}^{n-1}\right)$. Otherwise, $Z \cap X_{\Gamma^{\prime}}^{n-1}$ is an effective $k$ cycle with class $\alpha^{\prime}=$ $a H_{k}-\sum_{i=1}^{r} b_{i}^{\prime} E_{i, k}$, where $b_{i}^{\prime} \geq b_{i}$. Consequently, the class $\alpha=a H_{k}-\sum_{i=1}^{r} b_{i} E_{i, k}$ is effective. Then the cone $C(\alpha)$ is an effective class in $X_{\Gamma}^{n}$ and, since $b_{0} \leq a,[Z]$ is in the span of $E_{0, k+1}$ and $C(\alpha)$.

Let $Z$ be a cycle that generates an extremal ray of $\overline{C E f f}_{k}\left(X_{\Gamma^{\prime}}^{n-1}\right)$. Suppose $[Z]=\alpha+\beta$ in $\overline{\operatorname{Eff}}_{k+1}\left(X_{\Gamma}^{n}\right)$. Since $b_{0} \leq a$ holds on $\overline{\operatorname{Eff}}_{k+1}\left(X_{\Gamma}^{n}\right)$ and $b_{0}=a$ on $\mathrm{CEff}{ }_{k}\left(X_{\Gamma^{\prime}}^{n-1}\right)$, we must have that both $\alpha$ and $\beta$ satisfy $b_{0}=a$. We can perturb $\alpha$ and $\beta$ by $\epsilon H_{k+1}$ to obtain rational effective classes. Since the coefficient of $E_{0, k+1}$ of any class contained in $\overline{\mathrm{Eff}}_{k+1}\left(X_{\Gamma^{\prime}}^{n-1}\right)$ is 0 , the coefficients of any component of the class contained in $\overline{\operatorname{Eff}}_{k+1}\left(X_{\Gamma^{\prime}}^{n-1}\right)$ are bounded by $\epsilon$. Taking cones over the classes of the hyperplane sections of the remaining subvarieties and letting $\epsilon$ tend to zero, we see

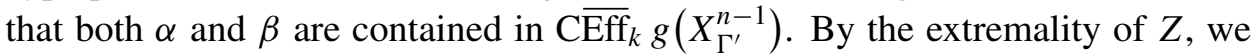
conclude that they are both proportional to $[Z]$.

Corollary 6.5. Let $\Gamma$ be a set of points $\left\{q_{1}, \ldots, q_{9}, p_{1}, \ldots, p_{s-1}\right\}$ such that the $q_{i}$ are general points in a plane $P \subset \mathbb{P}^{n}$ and the $p_{i}$ are linearly general points with span disjoint from $P$. Then $\overline{\mathrm{Eff}}_{k}\left(X_{\Gamma}^{n}\right)$ is not finitely generated for $k \leq s$ and is linearly generated for $k>s$.

Proof. When $r \geq 9$, the blowup of $\mathbb{P}^{2}$ at $r$ general points has infinitely many $(-1)$-curves, which span extremal rays of the effective cone of curves. Applying Proposition $6.4(k-1)$-times, the cones over the classes of $(-1)$-curves with vertex 
$p_{1}, \ldots, p_{k-1}$ provide infinitely extremal rays of $\overline{\operatorname{Eff}}_{k}\left(X_{\Gamma}^{n}\right)$ for $k \leq s$. The linear generation of $\overline{\operatorname{Eff}}_{k}\left(X_{\Gamma}^{n}\right)$ for $k>s$ follows from Proposition 6.1.

Corollary 6.6. (1) Linear generation of $\overline{\mathrm{Eff}}_{k}\left(X_{\Gamma}^{n}\right)$ is not closed in smooth families.

(2) Finite generation of $\overline{\operatorname{Eff}}_{k}\left(X_{\Gamma}^{n}\right)$ is not closed in smooth families.

Proof. Let $n \geq k+8$. Take a general smooth curve $B$ in $\left(\mathbb{P}^{n}\right)^{k+8}$ which avoids all the diagonals and contains a point $0 \in B$ where 9 of the points are general points in a plane $P$ and the remaining points are in linearly general position with span not intersecting $P$. Such curves exist by Bertini's Theorem since the diagonals have codimension $n \geq 2$. Consider the family $\mathcal{X} \rightarrow B$, where $\mathcal{X}_{b}$ is the blowup of $\mathbb{P}^{n}$ in the $k+8$ points $\Gamma_{b}$ parametrized by $b \in B$. If the points in $\Gamma_{b}$ are in linearly general position, then by Lemma 3.3 the cone $\overline{\operatorname{Eff}}_{k}\left(X_{\Gamma_{b}}\right)$ is linearly generated. In particular, the cone is finite. However, by Corollary $6.5, \overline{\operatorname{Eff}}_{k}\left(X_{\Gamma_{0}}\right)$ is not finitely generated. In particular, the cone is not linearly generated.

Corollary 6.7. (1) Linear generation of $\overline{\operatorname{Eff}}_{k}\left(X_{\Gamma}^{n}\right)$ is not open in smooth families.

(2) Finite generation of $\overline{\mathrm{Eff}}_{k}\left(X_{\Gamma}^{n}\right)$ is not open in smooth families.

Proof. Let $B$ be a smooth curve parametrizing 9 general points in a plane $P$ becoming collinear at $0 \in B$. Let $\Gamma^{\prime}$ be $k-1$ points in general linear position in $\mathbb{P}^{n}$ whose span is disjoint from $P$. Let $\Gamma_{b}$ be the union of $\Gamma^{\prime}$ and the points parametrized by $b$. Consider the family $\mathcal{X} \rightarrow B$ obtained by blowing up $\mathbb{P}^{n}$ along $\Gamma_{b}$. When the points are collinear, $\overline{\operatorname{Eff}}_{k}\left(X_{\Gamma_{0}}\right)$ is linearly generated. However, for the general point of $B, \overline{\operatorname{Eff}}_{k}\left(X_{\Gamma_{b}}\right)$ is not finitely generated by Corollary 6.5 .

Remark 6.8. Corollary 6.7 is well-known for cones of divisors. For example, Castravet and Tevelev [2006] prove that the blowup of $\mathbb{P}^{n}$ at points on a rational normal curve is a Mori dream space. In particular, if we specialize a large number of points to lie on a rational normal curve, we see that being a Mori dream space is not an open condition.

One can ask for the finite/linear generation of $\overline{\operatorname{Eff}}_{k}\left(X_{\Gamma}^{n}\right)$ for $\Gamma$ any special set of points. Perhaps the following question is the most interesting among them.

Question 6.9. Let $\Gamma$ be a set of points contained in a rational normal curve in $\mathbb{P}^{n}$. Is $\overline{\operatorname{Eff}}_{k}\left(X_{\Gamma}^{n}\right)$ finitely generated? Is $\overline{\operatorname{Eff}}_{k}\left(X_{\Gamma}^{n}\right)$ generated by the classes of cones over secant varieties of projections of the rational normal curve?

By results of Castravet and Tevelev, the answer to Question 6.9 is affirmative for curves and divisors. The cone of curves $\overline{\operatorname{Eff}}_{1}\left(X_{\Gamma}^{n}\right)$ is generated by the class of the proper transform of the rational normal curve $n H_{1}-\sum_{i=1}^{r} E_{i, 1}$ and the classes of lines. The rational normal curve is cut out by quadrics. If a curve $B$ has positive intersection with a quadric containing the points, then by Lemma 2.6 the class of $B$ is spanned by the classes of lines. Otherwise, $B$ is contained in the base locus 
of all the quadrics containing the rational normal curve. Hence $B$ is a multiple of the rational normal curve. Castravet and Tevelev [2006] show that the classes of divisors are generated by linear spaces and codimension- 1 cones over secant varieties of the projection of the rational normal curve. We do not know whether $\overline{\operatorname{Eff}}_{2}\left(X_{\Gamma}^{n}\right)$ is generated by the classes of planes and cones over the rational normal curve with vertex a point of $\Gamma$.

\section{Acknowledgements}

We would like to thank Dawei Chen, Lawrence Ein, Mihai Fulger, Joe Harris, Brian Lehmann, Kristian Ranestad, and Kevin Tucker for helpful discussions on cones of higher codimension cycles. We also thank the referees for numerous helpful suggestions.

\section{References}

[Birkar et al. 2010] C. Birkar, P. Cascini, C. D. Hacon, and J. McKernan, "Existence of minimal models for varieties of log general type", J. Amer. Math. Soc. 23:2 (2010), 405-468. MR 2601039 Zbl 1210.14019

[Castravet and Tevelev 2006] A.-M. Castravet and J. Tevelev, "Hilbert's 14th problem and Cox rings", Compos. Math. 142:6 (2006), 1479-1498. MR 2278756 Zbl 1117.14048

[Chen and Coskun 2015] D. Chen and I. Coskun, "Extremal higher codimension cycles on moduli spaces of curves", Proc. Lond. Math. Soc. (3) 111:1 (2015), 181-204. MR 3404780 Zbl 06464911

[Ciliberto and Miranda 2011] C. Ciliberto and R. Miranda, "Homogeneous interpolation on ten points”, J. Algebraic Geom. 20:4 (2011), 685-726. MR 2819673 Zbl 1230.14040

[Coble 1982] A. B. Coble, Algebraic geometry and theta functions, American Mathematical Society Colloquium Publications 10, American Mathematical Society, Providence, R.I., 1982. MR 733252

[Coskun 2006] I. Coskun, "Degenerations of surface scrolls and the Gromov-Witten invariants of Grassmannians”, J. Algebraic Geom. 15:2 (2006), 223-284. MR 2199064 Zbl 1105.14072

[Coskun 2008] I. Coskun, "Gromov-Witten invariants of jumping curves", Trans. Amer. Math. Soc. 360:2 (2008), 989-1004. MR 2346480 Zbl 1222.14033

[Debarre et al. 2011] O. Debarre, L. Ein, R. Lazarsfeld, and C. Voisin, "Pseudoeffective and nef classes on abelian varieties", Compos. Math. 147:6 (2011), 1793-1818. MR 2862063 Zbl 1234.14008

[Debarre et al. 2013] O. Debarre, Z. Jiang, and C. Voisin, "Pseudo-effective classes and pushforwards", Pure Appl. Math. Q. 9:4 (2013), 643-664. MR 3263971 Zbl 1319.14010

[Dolgachev 2011] I. V. Dolgachev, "Cremona special sets of points in products of projective spaces", pp. 115-134 in Complex and differential geometry, edited by W. Ebeling et al., Springer Proc. Math. 8, Springer, Heidelberg, 2011. MR 2964472 Zbl 1227.14020

[de Fernex 2010] T. de Fernex, “On the Mori cone of blow-ups of the plane”, preprint, 2010. arXiv 1001.5243

[Fulger and Lehmann 2014a] M. Fulger and B. Lehmann, "Kernels of numerical pushforwards", preprint, 2014. arXiv 1407.6455

[Fulger and Lehmann 2014b] M. Fulger and B. Lehmann, "Positive cones of dual cycle classes", preprint, 2014. arXiv 1408.5154 
[Fulton 1998] W. Fulton, Intersection theory, 2nd ed., Ergebnisse der Mathematik (3) 2, Springer, Berlin, 1998. MR 1644323 Zbl 0885.14002

[Gimigliano 1987] A. Gimigliano, On linear systems of plane curves, Ph.D. thesis, Queen's University, Ann Arbor, MI, 1987. MR 2635606

[Harbourne 1986] B. Harbourne, "The geometry of rational surfaces and Hilbert functions of points in the plane”, pp. 95-111 in Proceedings of the 1984 Vancouver conference in algebraic geometry, edited by J. Carrell et al., CMS Conf. Proc. 6, Amer. Math. Soc., Providence, RI, 1986. MR 846019 Zbl 0611.14002

[Harris 1995] J. Harris, Algebraic geometry, Graduate Texts in Mathematics 133, Springer, New York, 1995. MR 1416564

[Hirschowitz 1989] A. Hirschowitz, "Une conjecture pour la cohomologie des diviseurs sur les surfaces rationnelles génériques”, J. Reine Angew. Math. 397 (1989), 208-213. MR 993223

[Lazarsfeld 2004] R. Lazarsfeld, Positivity in algebraic geometry I, Ergebnisse der Mathematik (3) 48, Springer, Berlin, 2004. MR 2095471 Zbl 1093.14501

[Li 2015] Q. Li, "Pseudo-effective and nef cones on spherical varieties", Math. Z. 280:3-4 (2015), 945-979. MR 3369360 Zbl 06468909

[Mukai 2004] S. Mukai, "Geometric realization of $T$-shaped root systems and counterexamples to Hilbert's fourteenth problem", pp. 123-129 in Algebraic transformation groups and algebraic varieties, edited by V. L. Popov, Encyclopaedia Math. Sci. 132, Springer, Berlin, 2004. MR 2090672 Zbl 1108.13300

[Nagata 1959] M. Nagata, “On the 14-th problem of Hilbert”, Amer. J. Math. 81 (1959), 766-772. MR 0105409 Zbl 0192.13801

[Ottem 2011] J. C. Ottem, "On the Cox ring of $\mathbb{P}^{2}$ blown up in points on a line", Math. Scand. 109:1 (2011), 22-30. MR 2869458 Zbl 1221.14007

[Ottem 2015] J. C. Ottem, "Nef cycles on some hyperkähler fourfolds", 2015. arXiv 1505.01477

Communicated by János Kollár

Received 2016-03-15 Revised 2016-07-11 Accepted 2016-08-21

coskun@math.uic.edu

Department of Mathematics, Statistics, and Computer Science, University of Illinois at Chicago, Chicago, IL 60607, United States

jdl@uic.edu

Department of Mathematics, Statistics, and Computer Science, University of Illinois at Chicago, Chicago, IL 60607, United States

johnco@math.uio.no

Department of Mathematics, University of Oslo, Blindern, 0316 Oslo, Norway 


\section{Algebra \& Number Theory}

msp.org/ant

\section{EDITORS}

MANAGING EDITOR

Bjorn Poonen

Massachusetts Institute of Technology

Cambridge, USA

\author{
EDITORIAL BOARD CHAIR \\ David Eisenbud \\ University of California \\ Berkeley, USA
}

BOARD OF EDITORS

$\begin{aligned} \text { Dave Benson } & \text { University of Aberdeen, Scotland } & \text { Susan Montgomery } & \text { University of Southern California, USA } \\ \text { Richard E. Borcherds } & \text { University of California, Berkeley, USA } & \text { Shigefumi Mori } & \text { RIMS, Kyoto University, Japan } \\ \text { John H. Coates } & \text { University of Cambridge, UK } & \text { Raman Parimala } & \text { Emory University, USA } \\ \text { J-L. Colliot-Thélène } & \text { CNRS, Université Paris-Sud, France } & \text { Jonathan Pila } & \text { University of Oxford, UK } \\ \text { Brian D. Conrad } & \text { Stanford University, USA } & \text { Anand Pillay } & \text { University of Notre Dame, USA } \\ \text { Hélène Esnault } & \text { Freie Universität Berlin, Germany } & \text { Victor Reiner } & \text { University of Minnesota, USA } \\ \text { Hubert Flenner } & \text { Ruhr-Universität, Germany } & \text { Peter Sarnak } & \text { Princeton University, USA } \\ \text { Sergey Fomin } & \text { University of Michigan, USA } & \text { Joseph H. Silverman } & \text { Brown University, USA } \\ \text { Edward Frenkel } & \text { University of California, Berkeley, USA } & \text { Michael Singer } & \text { North Carolina State University, USA } \\ \text { Andrew Granville } & \text { Université de Montréal, Canada } & \text { Vasudevan Srinivas } & \text { Tata Inst. of Fund. Research, India } \\ \text { Joseph Gubeladze } & \text { San Francisco State University, USA } & \text { J. Toby Stafford } & \text { University of Michigan, USA } \\ \text { Roger Heath-Brown } & \text { Oxford University, UK } & \text { Ravi Vakil } & \text { Stanford University, USA } \\ \text { Craig Huneke } & \text { University of Virginia, USA } & \text { Michel van den Bergh } & \text { Hasselt University, Belgium } \\ \text { Kiran S. Kedlaya } & \text { Univ. of California, San Diego, USA } & \text { Marie-France Vignéras } & \text { Université Paris VII, France } \\ \text { János Kollár } & \text { Princeton University, USA } & \text { Kei-Ichi Watanabe } & \text { Nihon University, Japan } \\ \text { Yuri Manin } & \text { Northwestern University, USA } & \text { Efim Zelmanov } & \text { University of California, San Diego, USA } \\ \text { Philippe Michel } & \text { École Polytechnique Fédérale de Lausanne } & \text { Shou-Wu Zhang } & \text { Princeton University, USA }\end{aligned}$

PRODUCTION

production@msp.org

Silvio Levy, Scientific Editor

See inside back cover or msp.org/ant for submission instructions.

The subscription price for 2016 is US $\$ 290$ /year for the electronic version, and $\$ 485 /$ year (+\$55, if shipping outside the US) for print and electronic. Subscriptions, requests for back issues and changes of subscribers address should be sent to MSP.

Algebra \& Number Theory (ISSN 1944-7833 electronic, 1937-0652 printed) at Mathematical Sciences Publishers, 798 Evans Hall \#3840, c/o University of California, Berkeley, CA 94720-3840 is published continuously online. Periodical rate postage paid at Berkeley, CA 94704, and additional mailing offices.

ANT peer review and production are managed by EditFLow ${ }^{\circledR}$ from MSP.

\section{PUBLISHED BY}

- mathematical sciences publishers

nonprofit scientific publishing

http://msp.org/

() 2016 Mathematical Sciences Publishers 


\section{Algebra \& Number Theory}

Volume $10 \quad$ No. $9 \quad 2016$

Torsion pour les variétés abéliennes de type I et II

MARC HINDRY and NICOLAS RATAZZI

Galois-generic points on Shimura varieties

ANNA CADORET and ARNO KRET

Extremality of loci of hyperelliptic curves with marked Weierstrass points

DAWEI CHEN and NiCOLA TARASCA

$\bar{\Re}_{15}$ is of general type

GREGOR BRUNS

A vanishing theorem for weight-one syzygies

LAWRENCE EIN, ROBERT LAZARSFELD and DAVID YANG

1983

Effective cones of cycles on blowups of projective space

IZZET COSKUN, JOHN LESIEUTRE and JOHN CHRISTIAN OTTEM

Cluster algebras and category $\mathrm{O}$ for representations of Borel subalgebras of quantum affine algebras

DAVID HERNANDEZ and BERNARD LECLERC 